\title{
Analysis of an SVEIR model with age-dependence vaccination, latency and relapse
}

\author{
Jinliang Wang, Xiu Dong, Hongquan Sun*
}

School of Mathematical Science, Heilongjiang University, Harbin 150080, China.

Communicated by A. Atangana

\begin{abstract}
In this paper, we propose an epidemic model with age-dependence vaccination, latency and relapse. We derive the positivity and boundedness of solutions and find the basic reproduction number. Asymptotic smoothness, the existence of global compact attractor and uniform persistence of the model are investigated. By constructing Lyapunov functionals, we establish global stability of the equilibria in a threshold type. (C)2017 All rights reserved.
\end{abstract}

Keywords: Vaccination age, latency age, relapse age, global stability, Lyapunov function.

2010 MSC: 34D30, 92D30.

\section{Introduction}

There is growing interest in the dynamics of epidemic model with age structure (see, e.g., $[1,2,4,11$, $13,14,16,17,23-26,28]$ and references cited therein). In general, the average age at which individuals become infected is largely determined by the prevalence of infection in the population. Some evidences can be found in recent studies that the probability of becoming infected is dependent on age of the host $[5,6]$. It becomes more reasonable to study the important consequences of mathematical model with age structure.

In this paper, we divide the population into five classes: susceptible (those who are capable of contracting the disease); vaccinees (those who are vaccinated to defeat disease); exposed (those who are infected but not yet infectious); infectious (those who are infected and capable of transmitting the disease); and recovered (those who are permanently immune). Denoted by $S(t)$ the number of susceptible individuals at time $t$. Without infection, $S(t)$ is governed by the equation

$$
\frac{\mathrm{d} S(\mathrm{t})}{\mathrm{dt}}=(1-\mathrm{p}) \Lambda-\mu \mathrm{S}(\mathrm{t})
$$

where a fraction $1-p$ stands for the vaccination given immediately after birth or in newborn. $\mu$ is natural death rate. Susceptible individuals can come into contacting with infectious individuals and become new infected individuals at a rate $\beta$.

\footnotetext{
${ }^{*}$ Corresponding author

Email addresses: jinliangwang@hlju.edu.cn (Jinliang Wang), ngxiaoxiu@163.com (Xiu Dong), sunhongquan@hlju.edu.cn (Hongquan Sun)

doi:10.22436/jnsa.010.07.31
} 
The vaccinated class is structured by the age of vaccination a (i.e., the newly vaccinated individuals enter the vaccinated class with vaccine-age is zero), and the density of vaccinated individuals with respect to the age of vaccination $a$ at time $t$ is assumed to be $V(t, a)$. Then the total number of vaccinated individuals at time $t$ is given by $\int_{0}^{\infty} V(t, a) d a$. In our model, we consider a scenario that the vaccine is imperfect, which means that the vaccinated individuals still have the possibility to be infected. Let $I(t)$ denote the density of infectious individuals at time $t$. Throughout the paper, we always assume that vaccinated individuals can be infected at a rate $I(t) \int_{0}^{\infty} h(a) V(t, a) d a$, after contacting with infectious ones, where $h(a)$ stands for probability of infection with age-dependence vaccination. Let a fraction $p$ of vaccinated new-borns enter vaccinated class, which yields a boundary condition $V(t, 0)=p \wedge$.

Let $\alpha(a)$ be the rate at which the vaccine wanes, which is a nonnegative bounded function of vaccineage $a$. Thus vaccinated individuals eventually become susceptible again by a rate $\int_{0}^{\infty} \alpha(a) V(t, a) d a$. Under these assumptions, susceptible and vaccinated individuals are governed by the following equations:

$$
\left\{\begin{array}{l}
\frac{d S(t)}{d t}=(1-p) \wedge-\mu S(t)-\beta S(t) I(t)+\int_{0}^{\infty} \alpha(a) V(t, a) d a \\
V(t, 0)=p \wedge \\
\left(\frac{\partial}{\partial t}+\frac{\partial}{\partial a}\right) V(t, a)=-(\mu+\alpha(a)) V(t, a)-I(t) h(a) V(t, a) .
\end{array}\right.
$$

After infection, susceptible and vaccinated individuals enter into a stage where individuals are infected with the disease but not yet infective. This stage of the disease is often called latent stage. Waiting time for individuals in latent class varies from one to one, which may depend on the types of infectious diseases and individuals' status. Latent tuberculosis may take months, years or even decades to become infectious, thus, it would develop into a deadly disease if not treated or incompletely treated. Furthermore, the risk per unit time of activation appears to be higher in the early stages of latency than in later stages. McCluskey [16] investigated a model of disease transmission with continuous age-structure SEIR model for latently infected individuals and for infectious individuals. Liu et al. [11] paid attentions to an SEIR epidemic model with age dependent latency and relapse and proved that the threshold property can be preserved by defining the basic reproduction number. Here recurrent phenomenon are formulated as partial differential equations with age-dependent relapse rate (or varying relapse rate) instead of a constant relapse period and a general relapse distribution. Therefore, it is necessary to incorporate the duration age into modeling. In this paper, we structured exposed class as the age of duration (i.e., the duration time spent in this class). Let the density of exposed individuals with latency-age $a$ at time $t$ be $E(t, a)$, then the total number of exposed individuals at time $t$ is given by $\int_{0}^{\infty} E(t, a) d a$. The removal rate from latent class is given by the function $\sigma(a)$. Thus, the total rate at which individuals progress into the infectious class alive reads $\int_{0}^{\infty} \sigma(a) e(t, a) d a$

$$
\left\{\begin{array}{l}
E(t, 0)=\beta S(t) I(t)+I(t) \int_{0}^{\infty} h(a) V(t, a) d a, \\
\left(\frac{\partial}{\partial t}+\frac{\partial}{\partial a}\right) E(t, a)=-(\mu+\sigma(a)) E(t, a) .
\end{array}\right.
$$

In a recent study, van den Driessche et al. [20], van den Driessche and Zou [21] proposed the relapse takes the form of a constant relapse period and a general relapse distribution, respectively. Individuals come into the removed class after recovery (that may be natural or due to treatment). Let $k$ be the recovery rate from the infectious class. The density of individuals in removed class is denoted by $R(t, b)$, where $b$ is referred as relapse age for short. Thus the total number of removed individuals at time $t$ is given by $\int_{0}^{\infty} R(t, b) d a$. Let $\gamma(b)$ be the age-dependent relapse rate in removed class. The total rate at which individuals relapse into the infectious class is given by $\int_{0}^{\infty} \gamma(b) R(t, b) d b$. Thus infectious and recovered individuals are governed by the following equations:

$$
\left\{\begin{array}{l}
\frac{d I(t)}{d t}=\int_{0}^{\infty} \sigma(a) E(t, a) d a-(\mu+k) I(t)+\int_{0}^{\infty} \gamma(b) R(t, b) d b, \\
R(t, 0)=k I(t), \\
\left(\frac{\partial}{\partial t}+\frac{\partial}{\partial a}\right) R(t, b)=-(\gamma(b)+\mu) R(t, b) .
\end{array}\right.
$$


In this paper, the model to be studied takes the following form,

$$
\left\{\begin{array}{l}
\frac{d S(t)}{d t}=(1-p) \wedge-\mu S(t)-\beta S(t) I(t)+\int_{0}^{\infty} \alpha(a) V(t, a) d a \\
V(t, 0)=p \wedge \\
\left(\frac{\partial}{\partial t}+\frac{\partial}{\partial a}\right) V(t, a)=-(\mu+\alpha(a)) V(t, a)-I(t) h(a) V(t, a), \\
E(t, 0)=\beta S(t) I(t)+I(t) \int_{0}^{\infty} h(a) v(t, a) d a, \\
\left(\frac{\partial}{\partial t}+\frac{\partial}{\partial a}\right) E(t, a)=-(\mu+\sigma(a)) E(t, a), \\
\frac{d I(t)}{d t}=\int_{0}^{\infty} \sigma(a) E(t, a) d a-(\mu+k) I(t)+\int_{0}^{\infty} \gamma(b) R(t, b) d b \\
R(t, 0)=k I(t), \\
\left(\frac{\partial}{\partial t}+\frac{\partial}{\partial a}\right) R(t, b)=-(\gamma(b)+\mu) R(t, b) .
\end{array}\right.
$$

The initial conditions for model equations (1.4) are assumed to be

$$
S(0)=S_{0}>0, V(0, a)=V_{0}(a), E(0, a)=E_{0}(a), I(0)=I_{0}>0, R(0, b)=R_{0}(b) .
$$

To be mathematically tractable, we make the following assumption on functions of (1.1), (1.2), (1.3).

\section{Assumption 1.1.}

(i) $\alpha(\cdot), h(\cdot), \sigma(\cdot), \gamma(\cdot)$ are non-negative and belong to $L_{+}^{\infty}(0, \infty)$ with essential upper bounds $\bar{\alpha}, \bar{h}, \bar{\sigma}, \bar{\gamma} \in$ $(0, \infty)$, respectively;

(ii) $h(a)$ is Lipschitz continuous on $\mathbb{R}^{+}$with Lipschitz coefficients $M_{h}$;

(iii) there exists positive constant $\mu_{0} \in[0, \mu]$ such that $\alpha(a), \sigma(a), \gamma(b) \geqslant \mu_{0}$ for $a, b \geqslant 0$.

Denote by

$$
\varepsilon(a)=\sigma(a)+\mu, \quad \Gamma(a)=e^{-\int_{0}^{a} \varepsilon(\tau) d \tau},
$$

and

$$
\eta(b)=\gamma(b)+\mu, \quad \Upsilon(b)=e^{-\int_{0}^{b} \eta(\tau) d \tau} .
$$

It follows from (iii) of Assumption 1.1 that

$$
0 \leqslant \Gamma(a) \leqslant e^{-\left(\mu_{0}+\mu\right) a} \leqslant 1, \quad \frac{d \Gamma(a)}{d a}=-\varepsilon(a) \Gamma(a),
$$

and

$$
0 \leqslant \Upsilon(b) \leqslant e^{-\left(\mu_{0}+\mu\right) b} \leqslant 1, \quad \frac{d \Upsilon(b)}{d b}=-\eta(b) \Upsilon(b) .
$$

In this paper, we firstly employ the method developed by $[10,12,18,27]$ for age-dependent models, to reformulate the system as a system of Volterra integral equations. We verify the existence and uniqueness of solutions. Further we get a continuous solution semiflow $\{\Phi\}_{t \geqslant 0}$ on $X_{0+}$ generated by system (1.4). Furthermore, according to the similar approach in $[9,15]$, we shall establish the uniform persistence and the existence of a compact global attractor of system (1.4), which we can make use of the invariance principle. On the other hand, the uniform persistence of solutions ensures the each solution bounded below by some positive constants, which provide the well-posedness of Lyapunov function (defined to prove the global attractivity of equilibria). Finally, we show the global stability of equilibria by proper Volterra-type Lyapunov functions in terms of the basic reproduction number.

This paper is organized as follows. Section 2 is devoted to the well-posedness of the model. In Section 3, we investigate asymptotic smoothness of the semi-flow generated by the system. Section 4 contributes to the uniform persistence of system. In Section 5, the global stability of equilibria is proved by constructing candidate Lyapunov functions. 


\section{State space and well-posedness}

\subsection{Integrated semigroup formulation}

System (1.4) is a hybrid system of ordinary and partial differential equations. Taking into account the boundary conditions, we first rewrite is as a semilinear Cauchy problem. Following the method of [18], we first set the state space,

$$
X=\mathbb{R} \times \mathbb{R} \times \mathrm{L}^{1}((0, \infty), \mathbb{R}) \times \mathbb{R} \times \mathrm{L}^{1}((0, \infty), \mathbb{R}) \times \mathbb{R} \times \mathbb{R} \times \mathrm{L}^{1}((0, \infty), \mathbb{R}),
$$

and

$$
x_{+}=\mathbb{R}_{+} \times \mathbb{R}_{+} \times \mathrm{L}_{+}^{1}((0, \infty), \mathbb{R}) \times \mathbb{R}_{+} \times \mathrm{L}_{+}^{1}((0, \infty), \mathbb{R}) \times \mathbb{R}_{+} \times \mathbb{R}_{+} \times \mathrm{L}_{+}^{1}((0, \infty), \mathbb{R}),
$$

endowed with the usual product norm, and set

$$
X_{0}=\mathbb{R} \times\{0\} \times \mathrm{L}^{1}((0, \infty), \mathbb{R}) \times\{0\} \times \mathrm{L}^{1}((0, \infty), \mathbb{R}) \times \mathbb{R} \times\{0\} \times \mathrm{L}^{1}((0, \infty), \mathbb{R}),
$$

and

$$
x_{0+}=x_{0} \cap x_{+}
$$

We consider the linear operator $\mathcal{A}: \operatorname{Dom}(\mathcal{A}) \subset X \rightarrow X$ defined by

$$
\mathcal{A}\left(\begin{array}{c}
S \\
\left(\begin{array}{l}
0 \\
V
\end{array}\right) \\
\left(\begin{array}{c}
0 \\
E
\end{array}\right) \\
I \\
\left(\begin{array}{l}
0 \\
R
\end{array}\right)
\end{array}\right)=\left(\begin{array}{c}
-\mu S+\int_{0}^{\infty} \alpha(a) V(t, a) d a \\
-V(0) \\
-V^{\prime}-(\mu+\alpha(a)) V
\end{array}\right)
$$

with

$$
\operatorname{Dom}(\mathcal{A})=\mathbb{R} \times 0 \times 0 \times \mathrm{W}^{1,1}\left((0, \infty), \mathbb{R}^{2}\right) \times \mathbb{R} \times 0 \times \mathrm{W}^{1,1}((0, \infty), \mathbb{R}),
$$

where $W^{1,1}$ is a Sobolev space. Note that $\overline{\operatorname{Dom}(\mathcal{A})}=X_{0}$ is not dense in $X$.

Define nonlinear operator $\mathcal{F}: \overline{\operatorname{Dom}(\mathcal{A})} \rightarrow X$ by

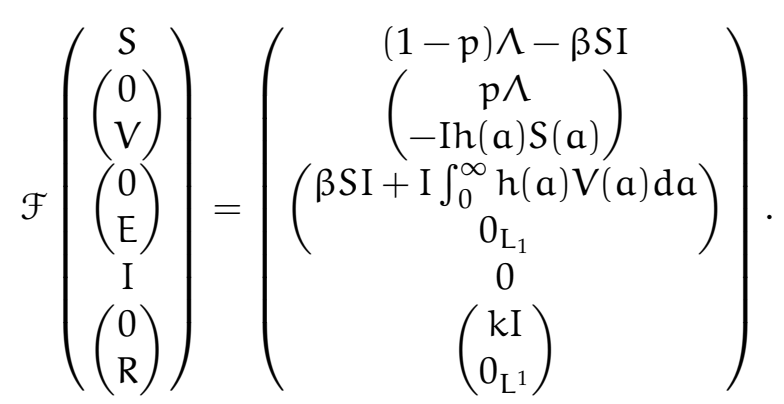

Then by identifying $u(t)=\left(S(t),\left(\begin{array}{c}0 \\ V(t, \cdot)\end{array}\right),\left(\begin{array}{c}0 \\ E(t, \cdot)\end{array}\right), I(t),\left(\begin{array}{c}0 \\ R(t, \cdot)\end{array}\right)\right)^{T}$, we can rewrite the system (1.4) with initial conditions (1.5) as the following abstract Cauchy problem

$$
\frac{d u(t)}{d t}=\mathcal{A u}(t)+\mathcal{F}(u(t))
$$

for $t \geqslant 0$ and $u(0)=u_{0} \in X_{0+}$.

We restrict our attention on the state space for system (1.4) to

$$
y=\mathbb{R}_{+} \times \mathrm{L}_{+}^{1}(0, \infty) \times \mathrm{L}_{+}^{1}(0, \infty) \times \mathbb{R}_{+} \times \mathrm{L}_{+}^{1}(0, \infty),
$$


equipped with the norm

$$
\|(x, \varphi, \phi, y, \psi)\| y=|x|+\int_{0}^{\infty}|\varphi(a)| d a+\int_{0}^{\infty}|\phi(a)| d a+|y|+\int_{0}^{\infty}|\psi(a)| d a .
$$

Biologically, this norm is interpreted as the total population size.

The existence and uniqueness of the semiflow $\{\Phi\}_{t \geqslant 0}$ on $X_{0+}$ generated by system (1.4) can be assured by the results in $[10,12,18,27]$. Thus we get a continuous solution semi-flow $\Phi: \mathbb{R}_{+} \times y \rightarrow y$ defined by

$$
\Phi\left(t, X_{0}\right)=\Phi_{t}\left(X_{0}\right):=(S(t), V(t, \cdot), E(t, \cdot), I(t), R(t, \cdot)), \quad t \geqslant 0, X_{0} \in y
$$

The precise result is the following proposition.

Proposition 2.1. For system (1.4), there exists a unique strongly continuous semiflow $\Phi: x_{0+} \rightarrow x_{0+\mathrm{t} \geqslant 0}$ such that for each $\mathrm{x}_{0} \in X_{0+}$, the operator $\mathrm{x} \in \mathrm{C}\left([0, \infty), X_{0+}\right)$ defined by $\mathrm{x}=\Phi(\mathrm{t}) \mathrm{x}_{0}$ is a mild solution of (1.4), that is, it satisfies

$$
\int_{0}^{t} x(s) d s \in \operatorname{Dom}(\mathcal{A}), \text { and } x(t)=x_{0}+\mathcal{A} \int_{0}^{t} x(s) d s+\int_{0}^{t} \mathcal{F}(x(s)) d s, \quad \forall t \geqslant 0 .
$$

\subsection{Volterra formulation}

For ease of notations, we introduce,

$$
\zeta(\mathrm{a})=\alpha(\mathrm{a})+\mu, \quad \Delta(\mathrm{a})=\mathrm{e}^{-\int_{0}^{\mathrm{a}} \zeta(\mathrm{r}) \mathrm{dr}},
$$

and

$$
n(t, \tau)=h(\tau) I(t-a+\tau)+\zeta(a), \quad \Omega(t, a)=e^{-\int_{0}^{a} n(t, \tau) d \tau} .
$$

Solving $V(t, a), E(t, a), R(t, b)$ in system (1.4) along the characteristic line $t-a=$ const. and $t-b=$ const. yields

$$
\begin{aligned}
& V(t, a)= \begin{cases}p \wedge \Omega(t, a), & \text { for } t>a \geqslant 0, \\
V_{0}(a-t) \frac{\Omega(t, a)}{\Omega(t, a-t)}, & \text { for } a \geqslant t \geqslant 0,\end{cases} \\
& E(t, a)= \begin{cases}{[\beta S(t-a) I(t-a)+H(t-a) I(t-a)] \Gamma(a),} & \text { for } t>a \geqslant 0, \\
E_{0}(a-t) \frac{\Gamma(a)}{\Gamma(a-t)}, & \text { for } a \geqslant t \geqslant 0,\end{cases}
\end{aligned}
$$

and

$$
R(t, b)= \begin{cases}k I(t-b) \Upsilon(b), & \text { for } t>b \geqslant 0, \\ R_{0}(b-t) \frac{\gamma(b)}{\gamma(b-t)}, & \text { for } b \geqslant t \geqslant 0 .\end{cases}
$$

Thus system (1.4) can be rewritten as the following Volterra-type equations

$$
\left\{\begin{array}{l}
\frac{d S(t)}{d t}=(1-p) \wedge-\mu S(t)-\beta S(t) I(t)+\int_{0}^{\infty} \alpha(a) V(t, a) d a, \\
V(t, a)= \begin{cases}p \wedge \Omega(t, a), & \text { for } t>a \geqslant 0, \\
V_{0}(a-t) \frac{\Omega(t, a)}{\Omega(t, a-t)}, & \text { for } a \geqslant t \geqslant 0,\end{cases} \\
E(t, a)= \begin{cases}{[\beta S(t-a) I(t-a)+H(t-a) I(t-a)] \Gamma(a),} & \text { for } t>a \geqslant 0, \\
E_{0}(a-t) \frac{\Gamma(a)}{\Gamma(a-t)}, & \text { for } a \geqslant t \geqslant 0,\end{cases} \\
\frac{d I(t)}{d t}=\int_{0}^{\infty} \begin{array}{ll}
\sigma(a) E(t, a) d a-(\mu+k) I(t)+\int_{0}^{\infty} \gamma(b) R(t, b) d b, \\
k I(t-b) \Upsilon(b), & \text { for } t>b \geqslant 0, \\
R_{0}(b-t) \frac{\gamma(b)}{\gamma(b-t)}, & \text { for } b \geqslant t \geqslant 0 .
\end{array}
\end{array}\right.
$$




\subsection{Boundness of semiflow}

The semiflow $\Phi\left(t, X_{0}\right)$ defined by (2.1) enjoys the nice properties as follows.

Proposition 2.2. Define

$$
\Xi:=\left\{x_{0}=\left(S_{0}, V_{0}(\cdot), E_{0}(\cdot), I_{0}, R_{0}(\cdot)\right) \in y \mid S_{0}+\left\|V_{0}(a)\right\|_{L^{1}}+\left\|E_{0}(a)\right\|_{L^{1}}+I_{0}+\left\|R_{0}(a)\right\|_{L^{1}} \leqslant \frac{\Lambda}{\mu}\right\} .
$$

Then, the following results hold.

(i) $\Xi$ is positively invariant for $\Phi$; i.e., $\Phi\left(t, x_{0}\right) \in \Xi$, for all $t \geqslant 0, x_{0} \in \Xi$.

(ii) $\Xi$ is point dissipative and $\Xi$ attracts all points in $y$.

(iii) There exist $\varepsilon_{1}, \varepsilon_{2}>0$ such that $\liminf _{t \rightarrow \infty} S(t) \geqslant \varepsilon_{1}, \liminf _{t \rightarrow \infty} \int_{0}^{\infty} V(t, a) \geqslant \varepsilon_{2}$, respectively.

Proof. Let

$$
N(t)=S(t)+\int_{0}^{\infty} V(t, a) d a+\int_{0}^{\infty} E(t, a) d a+I(t)+\int_{0}^{\infty} R(t, b) d b
$$

Adding the equations in (1.4) yields

$$
\begin{aligned}
\frac{d N(t)}{d t} & =\frac{d S(t)}{d t}+\frac{d}{d t} \int_{0}^{\infty} V(t, a) d a+\frac{d}{d t} \int_{0}^{\infty} E(t, a) d a+\frac{d I(t)}{d t}+\frac{d}{d t} \int_{0}^{\infty} R(t, b) d b \\
& =\Lambda-\mu\left(S(t)+\int_{0}^{\infty} V(t, a) d a+\int_{0}^{\infty} E(t, a) d a+I(t)+\int_{0}^{\infty} R(t, b) d b\right) \\
& =\Lambda-\mu N(t) .
\end{aligned}
$$

We integrate this differential inequality and obtain the a priori estimate,

$$
N(t)=\frac{\Lambda}{\mu}-e^{-\mu t}\left(\frac{\Lambda}{\mu}-N(0)\right) \leqslant \frac{\Lambda}{\mu}
$$

It follows that

$$
S(t)+\int_{0}^{\infty} V(t, a) d a+\int_{0}^{\infty} E(t, a) d a+I(t)+\int_{0}^{\infty} R(t, b) d b \leqslant \frac{\Lambda}{\mu}
$$

This implies that

$$
\lim \sup _{t \rightarrow \infty}\left\|\Phi_{t}\left(x_{0}\right)\right\|_{y}=\lim \sup _{t \rightarrow \infty} N(t) \leqslant \frac{\Lambda}{\mu},
$$

that is, $\Xi$ is positively invariant for $\Phi$, and further attracts each point in $\mathbb{Y}$. Thus (ii) directly follows. Next, we prove (iii). Since $I(t) \leqslant \frac{\Lambda}{\mu}$, it is easy to see from the first equation (1.1) that

$$
\frac{d S(t)}{d t} \geqslant(1-p) \Lambda-\left(\mu+\frac{\beta \Lambda}{\mu}\right) S(t)
$$

Recall that $\bar{h}, \bar{\alpha}$ are the essential upper bounds of $h(a), \alpha(a)$ for $a \geqslant 0$, respectively, from (i) of Assumption 1.1. We integrate this differential inequality and obtain the a priori estimate,

$$
\lim \inf _{t \rightarrow \infty} S(t) \geqslant \frac{(1-p) \Lambda}{\mu+\frac{\beta \Lambda}{\mu}}:=\varepsilon_{1} .
$$

Further, we have

$$
\frac{d}{d t} \int_{0}^{\infty} V(t, a) d a=-\int_{0}^{\infty}\left[\frac{\partial V(t, a)}{\partial a}+(h(a) I(t)+\alpha(a)+\mu) V(t, a)\right] d a
$$




$$
\begin{aligned}
& \geqslant-\left.V(t, a)\right|_{a=0} ^{a=\infty}-\left(\bar{h} \frac{\Lambda}{\mu}+\bar{\alpha}+\mu\right) \int_{0}^{\infty} V(t, a) d a \\
& =p \wedge-\left(\bar{h} \frac{\Lambda}{\mu}+\bar{\alpha}+\mu\right) \int_{0}^{\infty} V(t, a) d a,
\end{aligned}
$$

where we use $\left.V(t, a)\right|_{a=\infty}=0$. Thus we have

$$
\lim \inf _{t \rightarrow \infty} \int_{0}^{\infty} V(t, a) d a \geqslant \frac{p \wedge}{\bar{h} \frac{\Lambda}{\mu}+\bar{\alpha}+\mu}:=\varepsilon_{2} .
$$

This completes the proof.

The following proposition directly follows from Proposition 2.2.

Proposition 2.3. If $x_{0} \in y$ and $\left\|x_{0}\right\|_{y} \leqslant A$ for some constant $A \geqslant \frac{\Lambda}{\mu}$, then the following hold true for $t \geqslant 0$,

(i) $0 \leqslant S(t), \int_{0}^{\infty} V(t, a) d a, \int_{0}^{\infty} E(t, a) d a, I(t), \int_{0}^{\infty} R(t, b) d b \leqslant A$;

(ii) $\mathrm{H}(\mathrm{t}) \leqslant \overline{\mathrm{h}} \mathrm{A}$;

(iii) $E(t, 0) \leqslant(\beta+\bar{h}) A^{2}, R(t, 0) \leqslant k A$.

Using a similar argument as in [26, Proposition 4.1], we claim that the following proposition holds.

Proposition 2.4. Let $\mathrm{H}(\mathrm{t})=\int_{0}^{\infty} \mathrm{h}(\mathrm{a}) \mathrm{V}(\mathrm{t}, \mathrm{a})$ da. Then $\mathrm{H}(\mathrm{t})$ is Lipschitz continuous on $\mathbb{R}_{+}$with Lipschitz constant,

$$
\mathrm{L}_{\mathrm{H}}=\overline{\mathrm{h}}(\mu+\bar{\alpha}+\overline{\mathrm{h}} \mathrm{A}) \mathrm{A}+\mathrm{p} \wedge \overline{\mathrm{h}}+\mathrm{M}_{\mathrm{h}} \mathrm{A} .
$$

2.4. The basic reproductive number and equilibria

There always exists the disease-free equilibrium $E^{0}=\left(S^{0}, V^{0}(a), 0,0,0\right)$ satisfies

$$
S^{0}=\frac{\Lambda}{\mu}[(1-p)+p K], \quad V^{0}(a)=p \wedge \Omega(a), \quad E^{0}(a)=0, \quad I^{0}=0, \quad R^{0}(b)=0,
$$

where $\mathrm{K}=\int_{0}^{\infty} \alpha(\mathrm{a}) \Delta(\mathrm{a}) \mathrm{da}$ and $\Delta(\cdot)$ is defined in (2.2).

Define the basic reproduction number as,

$$
\mathfrak{R}_{0}=\frac{\left(\beta S^{0}+\int_{0}^{\infty} h(a) V^{0}(a) d a\right) \theta_{1}}{(\mu+k)-k \theta_{2}}
$$

where $\theta_{1}=\int_{0}^{\infty} \sigma(a) \Gamma(a) d a, \theta_{2}=\int_{0}^{\infty} \gamma(b) \Upsilon(b) d b$. Note that $1 /(\mu+k)$ is the average time in infectious class on the first pass, $k /(\mu+k)$ and $\theta_{2}$ are the probability of surviving the infectious class and entering the removed class alive, respectively. Thus the total average time in the infectious class not in the removed class (on multiple passes) is

$$
\frac{1}{\mu+k}\left[1+\frac{\delta \theta_{2}}{\mu+k}+\left(\frac{\delta \theta_{2}}{\mu+k}\right)^{2}+\cdots\right]=\frac{1}{\mu+k\left(1-\theta_{2}\right)} .
$$

Multiplying (2.6) by $\left(\beta S^{0}+\int_{0}^{\infty} h(a) V^{0}(a) d a\right) \theta_{1}$ gives $\mathfrak{R}_{0}$, which is the average number of new infections generated by a single newly infectious individual during the full infectious period. In what follows, we shall prove that $\mathfrak{R}_{0}$ serves as a threshold parameter for global stability of (1.4).

Denote

$$
n^{*}(a)=h(a) I^{*}+\zeta(a), \quad \Omega^{*}(a)=e^{-\int_{0}^{a} n^{*}(r) d r} .
$$


The equations for an equilibrium are obtained from (1.4) by setting the time derivatives equal to 0 with boundary conditions, that is, endemic equilibrium $\left(S^{*}, V^{*}(a), E^{*}(a), I^{*}, R^{*}(b)\right) \in y$ of (1.4) satisfies

$$
\left\{\begin{array}{l}
0=(1-p) \wedge-\mu S^{*}-\beta S^{*} I^{*}+\int_{0}^{\infty} \alpha(a) V^{*}(a) d a, \\
\frac{d V^{*}(a)}{d a}=-n^{*}(a) V^{*}(a), \\
\frac{d E^{*}(a)}{d a}=-\varepsilon(a) E^{*}(a), \\
0=\int_{0}^{\infty} \sigma(a) E^{*}(a) d a-(\mu+k) I^{*}+\int_{0}^{\infty} \gamma(b) R^{*}(b) d b \\
\frac{d R^{*}(b)}{d b}=-\eta(b) R^{*}(b), \\
E^{*}(0)=\beta S^{*} I^{*}+\int_{0}^{\infty} h(a) V^{*}(a) d a I^{*}, \\
R^{*}(0)=k I^{*}, V^{*}(0)=p \wedge .
\end{array}\right.
$$

From the second, third and fifth equations of (2.7), we obtain

$$
\begin{gathered}
\mathrm{V}^{*}(\mathrm{a})=\mathrm{V}^{*}(0) \Omega^{*}(\mathrm{a})=\mathrm{p} \wedge \Omega^{*}(\mathrm{a}), \\
\mathrm{E}^{*}(\mathrm{a})=\mathrm{E}^{*}(0) \Gamma(\mathrm{a})=\left(\beta \mathrm{S}^{*} \mathrm{I}^{*}+\int_{0}^{\infty} \mathrm{h}(\mathrm{a}) \mathrm{V}^{*}(\mathrm{a}) d a I^{*}\right) \Gamma(\mathrm{a}),
\end{gathered}
$$

and

$$
R^{*}(b)=R^{*}(0) \Upsilon(b)=k I^{*} \Upsilon(b) .
$$

One substitutes (2.8) into the first equation of (2.7), yields

$$
S^{*}=\frac{\Lambda}{\mu+\beta I^{*}}\left((1-p)+p \int_{0}^{\infty} \alpha(a) \Omega^{*}(a) d a\right)=\frac{\Lambda}{\mu+\beta I^{*}}\left[(1-p)+p K^{*}\right],
$$

where $K^{*}=\int_{0}^{\infty} \alpha(a) \Omega^{*}(a) d a$. Recall that $0<K^{*} \leqslant K \leqslant \int_{0}^{\infty} \alpha(a) e^{-\int_{0}^{a} \alpha(s) d s} d a=1$. Substituting (2.8), (2.9), (2.10) and $S^{0}$ into the fourth equation of (2.7) yields

$$
0=\beta S^{0} \theta_{1} \frac{\mu\left[(1-p)+p K^{*}\right]}{\left(\mu+\beta I^{*}\right)[(1-p)+p K]}+p \wedge \theta_{1} \int_{0}^{\infty} h(a) \Omega^{*}(a) d a+k \theta_{2}-(\mu+k) .
$$

Denote the right-hand side of (2.11) by $\Sigma\left(I^{*}\right)$. Some calculations yield

$$
\begin{aligned}
\Sigma^{\prime}\left(I^{*}\right)= & \beta S^{0} \theta_{1} \frac{\mu}{[(1-p)+p K]} \frac{-p\left(\mu+\beta I^{*}\right) \int_{0}^{\infty} \alpha(a) \Omega^{*}(a) d a \int_{0}^{a} h(r) d r-\beta\left[(1-p)+p K^{*}\right]}{\left(\mu+\beta I^{*}\right)^{2}} \\
& -p \wedge \theta_{1} \int_{0}^{\infty} h(a) \Omega^{*}(a) d a \int_{0}^{a} h(r) d r<0 .
\end{aligned}
$$

This implies that $\Sigma\left(I^{*}\right)$ monotonically decreases. Since

$$
\Sigma(0)=\beta S^{0} \theta_{1}+\theta_{1} \int_{0}^{\infty} h(a) V^{0}(a) d a-(\mu+k)+k \theta_{2}=\left(\mu+k-k \theta_{2}\right)\left(\Re_{0}-1\right),
$$

if $\mathfrak{R}_{0} \leqslant 1$, then $\Sigma(0) \leqslant 0$. So (2.11) has no positive real root. If $\mathfrak{R}_{0}>1$, then $\Sigma(0)>0$. This suggests that (2.11) admits a unique positive real root, denoted by $\mathrm{I}^{*}$. Thus a unique endemic equilibrium $\mathrm{E}^{*}=$ $\left(S^{*}(a), V^{*}(a), E^{*}(a), I^{*}, R^{*}(a)\right)$ exists under the condition $\Re_{0}>1$.

Theorem 2.5. Let $\mathfrak{R}_{0}$ be defined by (2.5), we have the following results.

(i) System (1.4) always admits a disease-free equilibrium $\mathrm{E}^{0}=\left(\mathrm{S}^{0}, \mathrm{~V}^{0}(\mathrm{a}), 0,0,0\right)$, which is defined by (2.4).

(ii) System (1.4) has a unique endemic equilibrium $\mathrm{E}^{*}$ when $\mathfrak{R}_{0}>1$. 


\section{Asymptotic smoothness of $\{\Phi(t)\}_{t} \geq 0$}

By Proposition 2.2, the semiflow is point-dissipative and $\Phi\left(\mathbb{R}_{+} \times B\right)$ is bounded for every bounded subset B of $y$. By [7, Theorem 3.4.6], the semiflow has a compact attractor of bounded sets if it is asymptotically smooth. To give the existence of compact attractor, we follow the approach in [22, Theorem 4.2 of Chapter IV].

Definition 3.1 ([19]). A set $A_{0}$ in $y$ is called a compact attractor of a set $B \subseteq X$ if $A_{0}$ is compact, invariant, and non-empty and $\Phi_{t}(B) \rightarrow A_{0}$ as $t \rightarrow \infty$. The last means that, for every open subset $U$ of $y$ with $A_{0} \subseteq \mathrm{U}$, there is some $r>0$ such that $\Phi_{\mathfrak{t}}(\mathrm{B}) \subseteq \mathrm{U}$ for all $\mathrm{t} \geqslant \mathrm{r}$ (i.e., $\Phi([r, \infty) \times \mathrm{B}) \subseteq \mathrm{U}$ ).

To this end, we first divide $\{\Phi(t)\}_{t} \geqslant 0$ into two parts,

$$
\Phi_{1}(t) x_{0}:=(0, \tilde{\varphi}(t, \cdot), \tilde{\phi}(t, \cdot), 0, \tilde{\psi}(t, \cdot))^{\top},
$$

and

$$
\Phi_{2}(t) x_{0}:=(S(t), \tilde{V}(t, \cdot), \tilde{E}(t, \cdot), I(t), \tilde{R}(t, \cdot))^{\top},
$$

That is to say, $\Phi(t) x_{0}=\Phi_{1}(t) x_{0}+\Phi_{2}(t) x_{0}$. Each component is defined by

$$
\begin{aligned}
& \tilde{\varphi}(t, \cdot)=\left\{\begin{array}{ll}
0, & \text { for } t>a \geqslant 0, \\
V(t, a), & \text { for } a \geqslant t \geqslant 0,
\end{array} \tilde{V}(t, \cdot)= \begin{cases}V(t, a), & \text { for } t>a \geqslant 0, \\
0, & \text { for } a \geqslant t \geqslant 0,\end{cases} \right. \\
& \tilde{\phi}(t, \cdot)=\left\{\begin{array}{ll}
0, & \text { for } t>a \geqslant 0, \\
E(t, a), & \text { for } a \geqslant t \geqslant 0,
\end{array} \quad \tilde{E}(t, \cdot)= \begin{cases}E(t, a) & \text { for } t>a \geqslant 0, \\
0, & \text { for } a \geqslant t \geqslant 0,\end{cases} \right.
\end{aligned}
$$

and

$$
\tilde{\psi}(t, \cdot)=\left\{\begin{array}{ll}
0, & \text { for } t>b \geqslant 0, \\
R(t, b), & \text { for } b \geqslant t \geqslant 0,
\end{array} \tilde{R}(t, \cdot)= \begin{cases}R(t, b), & \text { for } t>b \geqslant 0 \\
0, & \text { for } b \geqslant t \geqslant 0 .\end{cases}\right.
$$

Theorem $3.2([7,8])$. The semi-flow $\Phi: y \rightarrow y$ is asymptotically smooth if there are maps $\Phi_{1}, \Phi_{2}: \mathbb{R}_{+} \times y \rightarrow y$ s.t. $\Phi(\mathrm{t}, \mathrm{y})=\Phi_{1}(\mathrm{t}, \mathrm{y})+\Phi_{2}(\mathrm{t}, \mathrm{y})$, and the following hold for any bounded closed set $\Xi$ that is forward invariant under $\Phi$ :

(i) $\lim _{t \rightarrow \infty} \operatorname{diam} \Phi_{1}(t, \Xi)=0$;

(ii) there exists $t_{\Xi} \geqslant 0$ such that $\Phi_{2}(t, \Xi)$ has compact closure for each $t \geqslant t_{\Xi}$.

The rest of this section is spent on proving Theorem 3.2.

Recall that $\Phi_{1}(t)$ and $\Xi$ are defined by (3.1) and (2.3), respectively. It is sufficient to verify that there exists a function $z(\cdot, \cdot): \mathbb{R}_{+} \times \mathbb{R}_{+} \rightarrow \mathbb{R}_{+}$such that for any $r>0$,

$$
\lim _{t \rightarrow+\infty} z(t, r)=0
$$

and

$$
\left\|\Phi_{1}(\mathrm{t}) \mathrm{x}_{0}\right\|_{\mathrm{y}} \leqslant \mathrm{h}(\mathrm{t}, \mathrm{r}), \quad \forall \mathrm{x}_{0} \in \Xi,\left\|\mathrm{x}_{0}\right\|_{\mathrm{y}} \leqslant \mathrm{r}, \mathrm{t} \geqslant 0 .
$$

To this end, we rely on the Volterra formulations in (3.1) and (3.2). It is easy to see that for $x_{0} \in \Xi$ satisfying $\left\|x_{0}\right\|_{y} \leqslant r$,

$$
\begin{aligned}
\left\|\Phi_{1}\left(t, x_{0}\right)\right\|_{y}= & \|0\|+\|\tilde{\varphi}(t, \cdot)\|_{y}+\|\tilde{\phi}(t, \cdot)\|_{y}+\|0\|+\|\tilde{\Psi}(t, \cdot)\|_{y} \\
= & \int_{t}^{+\infty} V_{0}(a-t) e^{-\int_{0}^{t}[h(a-t+\tau) I(\tau)+\alpha(a-t+\tau)+\mu] d \tau} d a \\
& +\int_{t}^{+\infty} E_{0}(a-t) e^{-\int_{0}^{t}[\sigma(a-t+\tau) I(\tau)+\mu] d \tau} d a+\int_{t}^{+\infty} R_{0}(b-t) e^{-\int_{0}^{t}[\gamma(b-t+\tau)+\mu] d \tau} d b
\end{aligned}
$$




$$
\begin{aligned}
& \leqslant e^{-\mu_{0} t}\left(|0|+\int_{0}^{\infty}\left|V_{0}(\tau)\right| d \tau+\int_{0}^{\infty}\left|E_{0}(\tau)\right| d \tau+|0|+\int_{0}^{\infty}\left|R_{0}(\tau)\right| d \tau\right) \\
& \leqslant e^{-\mu_{0} t}\left\|x_{0}\right\| y,
\end{aligned}
$$

and this implies that (3.3) and (3.4) hold with $z(t, r)=r e^{-\mu_{0} t}$.

To verify condition (ii) of Theorem 3.2 holds true, it is sufficient to prove that for all $t \geqslant 0, \Phi_{2}(t)$ maps any bounded subsets of $\Xi$ into sets with compact closure in $y$. Recall that for $x_{0}$ in any bounded subset of $\Xi, I(t)$ remains in the compact set

$$
\left\{\psi \in \mathbb{R}_{+}^{n}: 0 \leqslant \psi \leqslant \frac{\Lambda}{\mu}\right\}
$$

for all $t \geqslant 0$. Therefore, in what follows, we shall show that $\tilde{V}(t, \cdot), \tilde{E}(t, \cdot)$ and $\tilde{R}(t, \cdot)$ remain in a precompact subset of $y$ which is independent of $x_{0}$. By the Fréchet-Kolmogorov theorem, it is sufficient to show that

$$
\begin{aligned}
& \tilde{V}(t, a):= \begin{cases}p \wedge e^{-\int_{0}^{a}\{h(\tau) I(t-a+\tau)+\alpha(\tau)+\mu\} d \tau,} & t-a>0, \\
0, & a-t>0,\end{cases} \\
& \tilde{E}(t, a):= \begin{cases}{[\beta S(t-a) I(t-a)+H(t-a) I(t-a)] e^{-\int_{0}^{a}\{\sigma(\tau)+\mu\} d \tau,}} & t-a>0, \\
0, & a-t>0,\end{cases}
\end{aligned}
$$

and

$$
\tilde{R}(t, b):=\left\{\begin{array}{lr}
k I(t-b) e^{-\int_{0}^{b}\{\gamma(\tau)+\mu\} d \tau,} & t-b>0, \\
0, & b-t>0,
\end{array}\right.
$$

satisfy the following conditions:

(i) The supremum of $\int_{0}^{+\infty} \tilde{\Theta}(t, a) d a$ for all initial data $x_{0} \in \Xi$ is finite.

(ii) $\lim _{h \rightarrow+\infty} \int_{h}^{+\infty} \tilde{\Theta}(t, a) d a=0$ uniformly in $x_{0} \in \Xi$.

(iii) $\lim _{h \rightarrow+0} \int_{0}^{+\infty}|\tilde{\Theta}(t, a+h)-\tilde{\Theta}(t, a)| d a=0$ uniformly in $x_{0} \in \Xi$.

(iv) $\lim _{h \rightarrow+0} \int_{0}^{h} \tilde{\Theta}(t, a) d a=0$ uniformly in $x_{0} \in \Xi$.

Recall that $\tilde{V}(t, a), \tilde{E}(t, a)$ and $\tilde{R}(t, b)$ are bounded from Propositions 2.2 and 2.3, and hence, (i), (ii) and (iv) immediately follow. We only need to verify $\tilde{V}(t, a), \tilde{E}(t, a)$ and $\tilde{R}(t, b)$ satisfy (iii), respectively. Since we shall consider $h \rightarrow+0$, we can assume that $h \in(0, t)$ without loss of generality. Then, from (3.5), we have

$$
\begin{aligned}
\int_{0}^{+\infty} & |\tilde{V}(t, a+h)-\tilde{V}(t, a)| d a \\
\quad & \int_{t-h}^{t}|0-\tilde{V}(t, a)| d a+\int_{0}^{t-h}|\tilde{V}(t, a+h)-\tilde{V}(t, a)| d a \\
\leqslant & p \wedge h+p \wedge \int_{0}^{t-h} \mid e^{-\int_{0}^{a+h}\{h(\tau) I(t-a-h+\tau)+\alpha(\tau)+\mu\} d \tau}-e^{-\int_{0}^{a}\{h(\tau) I(t-a+\tau)+\alpha(\tau)+\mu\} d \tau \mid} d a \\
\leqslant & p \wedge h+p \wedge \int_{0}^{t-h} \mid \int_{0}^{a+h}\{h(\tau) I(t-a-h+\tau)+\alpha(\tau)+\mu\} d \tau \\
& \quad-\int_{0}^{a}\{h(\tau) I(t-a+\tau)+\alpha(\tau)+\mu\} d \tau \mid d a,
\end{aligned}
$$

where we use the relation $\left|e^{-L_{1}(a)}-e^{-L_{2}(a)}\right| \leqslant\left|L_{1}(a)-L_{2}(a)\right|$. Thus, by a zero-trick, we have

$$
\int_{0}^{+\infty}|\tilde{V}(t, a+h)-\tilde{V}(t, a)| d a \leqslant p \wedge h+\mathcal{A}_{1}+\mathcal{B}_{1}
$$


where

$$
\begin{aligned}
\mathcal{A}_{1}:= & \mathrm{p} \wedge \int_{0}^{\mathrm{t}-\mathrm{h}} \mid \int_{0}^{\mathrm{a}+\mathrm{h}}\{\mathrm{h}(\tau) \mathrm{I}(\mathrm{t}-\mathrm{a}-\mathrm{h}+\tau)+\alpha(\tau)+\mu\} \mathrm{d} \tau \\
& -\int_{0}^{\mathrm{a}+\mathrm{h}}\{\mathrm{h}(\tau) \mathrm{I}(\mathrm{t}-\mathrm{a}+\tau)+\alpha(\tau)+\mu\} \mathrm{d} \tau \mid \mathrm{da} \\
& \leqslant \mathrm{p} \wedge \overline{\mathrm{h}} \int_{0}^{\mathrm{t}-\mathrm{h}} \int_{0}^{\mathrm{a}+\mathrm{h}}|\mathrm{I}(\mathrm{t}-\mathrm{a}-\mathrm{h}+\tau)-\mathrm{I}(\mathrm{t}-\mathrm{a}+\tau)| \mathrm{d} \tau \mathrm{d} \mathrm{a},
\end{aligned}
$$

and

$$
\begin{aligned}
\mathcal{B}_{1}:= & \mathrm{p} \wedge \int_{0}^{\mathrm{t}-\mathrm{h}} \mid \int_{0}^{\mathrm{a}+\mathrm{h}}\{\mathrm{h}(\tau) \mathrm{I}(\mathrm{t}-\mathrm{a}+\tau)+\alpha(\tau)+\mu\} \mathrm{d} \tau \\
& -\int_{0}^{\mathrm{a}}\{\mathrm{h}(\tau) \mathrm{I}(\mathrm{t}-\mathrm{a}+\tau)+\alpha(\tau)+\mu\} \mathrm{d} \tau \mid \mathrm{da} \\
& \leqslant \mathrm{p} \wedge(\bar{\alpha}+\mu) \mathrm{h}(\mathrm{t}-\mathrm{h})+\mathrm{p} \wedge \overline{\mathrm{h}} \int_{0}^{\mathrm{t}-\mathrm{h}} \int_{a}^{\mathrm{a}+\mathrm{h}}|\mathrm{I}(\mathrm{t}-\mathrm{a}+\tau)| \mathrm{d} \tau \mathrm{da},
\end{aligned}
$$

where $\bar{\sigma}, \bar{\gamma}, \overline{\mathrm{h}}$ are positive constants defined as in Assumption 1.1.

Since

$$
\left\|\frac{d}{d t} I(t)\right\| \leqslant\{\bar{\sigma} A+(\mu+k) A+\bar{\gamma} A\}=: M_{I}
$$

it follows that $I(t)$ is Lipschitz continuous on $\mathbb{R}_{+}$with Lipschitz coefficient $M_{I}$. Hence,

$$
\mathcal{A}_{1} \leqslant \mathrm{p} \wedge \overline{\mathrm{h}} \mathrm{M}_{\mathrm{I}} \mathrm{h} \int_{0}^{\mathrm{t}-\mathrm{h}}(\mathrm{a}+\mathrm{h}) \mathrm{da}=\mathrm{p} \wedge \overline{\mathrm{h}} \mathrm{M}_{\mathrm{I}} \mathrm{h}\left\{\frac{1}{2}(\mathrm{t}-\mathrm{h})^{2}+\mathrm{h}(\mathrm{t}-\mathrm{h})\right\} .
$$

Since $\|I(t)\| \leqslant A$ for all $t \geqslant 0$ from Proposition 2.3, we have

$$
\mathcal{B}_{1} \leqslant p \wedge(\bar{\alpha}+\mu) h(t-h)+p \wedge \bar{h} A h(t-h) .
$$

Consequently, it follows from (3.8), (3.9) and (3.10) that

$$
\begin{aligned}
\int_{0}^{+\infty}|\tilde{V}(t, a+h)-\tilde{V}(t, a)| d a \leqslant & p \wedge h+p \wedge \bar{h} M_{I} h\left\{\frac{1}{2}(t-h)^{2}+h(t-h)\right\} \\
& +p \wedge(\bar{\alpha}+\mu) h(t-h)+p \wedge \bar{h} A h(t-h),
\end{aligned}
$$

which approaches 0 as $\mathrm{h} \rightarrow+0$ in $\mathrm{x}_{0} \in \Xi$.

Similarly, from (3.6), we have

$$
\begin{aligned}
\int_{0}^{+\infty}|\tilde{E}(t, a+h)-\tilde{E}(t, a)| d a= & \int_{0}^{t}|E(t, a+h)-E(t, a)| d b \\
= & \int_{0}^{t-h}|E(t-a-h, 0) \Gamma(a+h)-E(t-a, 0) \Gamma(a)| d a \\
& +\int_{t-h}^{t}|E(t-a, 0) \Gamma(b)| d a \\
\leqslant & (\beta+\bar{h}) A^{2} h+\mathcal{A}_{2}+\mathcal{B}_{2},
\end{aligned}
$$

where

$$
\mathcal{A}_{2}=\int_{0}^{\mathrm{t}-\mathrm{h}} \mathrm{E}(\mathrm{t}-\mathrm{a}-\mathrm{h}, 0)|\Gamma(\mathrm{a}+\mathrm{h})-\Gamma(\mathrm{a})| \mathrm{da},
$$


and

$$
\mathcal{B}_{2}=\int_{0}^{t-h} \Gamma(a)|E(t-a-h, 0)-E(t-a, 0)| d a .
$$

Since

$$
\begin{aligned}
\int_{0}^{t-h}|\Gamma(a+h)-\Gamma(a)| d a & =\int_{0}^{t-h} \Gamma(a) d a-\int_{0}^{t-h} \Gamma(a+h) d a \\
& =\int_{0}^{t-h} \Gamma(a) d a-\int_{h}^{t} \Gamma(a) d a \\
& =\int_{0}^{t-h} \Gamma(a) d a-\int_{h}^{t-h} \Gamma(a) d a-\int_{t-h}^{t} \Gamma(a) d a \\
& =\int_{0}^{h} \Gamma(a) d a-\int_{t-h}^{t} \Gamma(a) d a \leqslant h,
\end{aligned}
$$

it follows from (iii) of Proposition 2.3, we have $\mathcal{A}_{2} \leqslant(\beta+\overline{\mathrm{h}}) \mathrm{A}^{2} \mathrm{~h}$. We rewrite $\mathcal{B}_{2}$ as

$$
\begin{aligned}
\mathcal{B}_{2}= & \int_{0}^{t-h} \mid[\beta S(t-a-h) I(t-a-h)+H(t-a-h) I(t-a-h)] \\
& -[\beta S(t-a) I(t-a)+H(t-a) I(t-a)] \mid \Gamma(a) d a \\
\leqslant & \int_{0}^{t-h}|[\beta S(t-a-h) I(t-a-h)-\beta S(t-a) I(t-a)]| \Gamma(a) d a \\
& +\int_{0}^{t-h}|[H(t-a-h) I(t-a-h)-H(t-a) I(t-a)]| \Gamma(a) d a .
\end{aligned}
$$

Since $S(t)$ and $I(t)$ are Lipschitz continuous on $\mathbb{R}_{+}$with Lipschitz constants $M_{S}=(1-p) \wedge+(\mu+\bar{\alpha}) A+$ $\beta A^{2}$ and $M_{I}=(\bar{\sigma}+\mu+k+\bar{\gamma}) A$, respectively. Recall that from Proposition 2.4, $\mathrm{H}(\mathrm{t})$ is Lipschitz continuous on $\mathbb{R}_{+}$with Lipschitz constant $L_{H}$, thus $S(t) I(t)$ and $H(t) I(t)$ are Lipschitz continuous with Lipschitz constants $M_{S I}=A M_{I}+A M_{S}$ and $M_{H I}=\bar{p} A M_{I}+A L_{H}$, respectively. Then taking $M=\beta M_{S I}+M_{H I}$, we get,

$$
\mathcal{B}_{2} \leqslant M h \int_{0}^{t-h} e^{-\left(\mu_{0}+\mu\right) a} d a \leqslant \frac{M h}{\mu_{0}+\mu} .
$$

Consequently,

$$
\int_{0}^{+\infty}|\tilde{E}(t, a+h)-\tilde{E}(t, a)| d a \leqslant 2(\beta+\bar{h}) A^{2} h+\frac{M h}{\mu_{0}+\mu}
$$

which approaches 0 as $\mathrm{h} \rightarrow+0$ in $x_{0} \in \Xi$.

Lastly, from (3.7), we have

$$
\begin{aligned}
\int_{0}^{+\infty}|\tilde{R}(t, b+h)-\tilde{R}(t, b)| d b= & \int_{0}^{t}|R(t, b+h)-R(t, b)| d b \\
= & \int_{0}^{t-h}|R(t-b-h, 0) \Upsilon(b+h)-R(t-b, 0) \Upsilon(b)| d b \\
& +\int_{t-h}^{t}|0-R(t-b, 0) \Upsilon(b)| d b \\
\leqslant & k \int_{0}^{t-h} I(t-b-h)|\Upsilon(b+h)-\Upsilon(b)| d b \\
& +k \int_{0}^{t-h} \Upsilon(b)|I(t-b-h)-I(t-b)| d b+\int_{t-h}^{t}|R(t-b, 0) \Upsilon(b)| d b .
\end{aligned}
$$


Similarly as above estimations, though in a different context, we get

$$
\int_{0}^{+\infty}|\tilde{R}(t, b+h)-\tilde{R}(t, b)| d b \leqslant\left(2 k A+\frac{k M_{I}}{\mu_{0}+\mu}\right) h,
$$

which converges uniformly to 0 as $h \rightarrow 0$. This completes to verify that $\tilde{V}(t, a), \tilde{E}(t, a)$ and $\tilde{R}(t, b)$ satisfy (iii), respectively.

\section{Uniform persistence for $\mathfrak{R}_{0}>1$}

This section is devoted to prove the uniform persistence of system (1.4) when $\mathfrak{R}_{0}>1$, which implies that there always exist infectious individuals if the infectivity initially presents.

We introduce the following notations to define the invariant sets of uniform persistence. Define

$$
\bar{a}:=\inf \left\{a: \int_{a}^{\infty} \sigma(a) d a=0\right\}, \bar{b}:=\inf \left\{b: \int_{b}^{\infty} \gamma(b) d b=0\right\}
$$

Obviously, $\overline{\mathrm{a}}, \overline{\mathrm{b}}>0$ due to $\sigma(\mathrm{a}), \gamma(\mathrm{b}) \in \mathrm{L}_{+}^{1}$. Further we define

$$
\begin{gathered}
\hat{X}:=L_{+}^{1} \times \mathbb{R}^{+} \times L_{+}^{1} \\
\hat{Y}:=\left\{(E(t, \cdot), I(t), R(t, \cdot))^{\top} \in \hat{X}: \int_{0}^{\bar{a}} E(t, a) d a>0 \text { or } I(t)>0 \text { or } \int_{0}^{\bar{b}} R(t, b) d b>0\right\},
\end{gathered}
$$

and

$$
Y:=\mathbb{R}_{+} \times L_{+}^{1} \times \hat{Y}, \quad \partial Y:=X \backslash Y, \quad \partial \hat{Y}:=\hat{X} \backslash \hat{Y} .
$$

Using the similar arguments in [11, Proposition 5.1], we get the following result.

Theorem 4.1. For the semi-flow $\Phi(t)_{t \geqslant 0}$ generated by system (1.4), the set $\partial Y$ is positively invariant, that is, $\Phi(t, \partial Y) \subset \partial Y$ for $\mathrm{t} \geqslant 0$.

Proof. Suppose that $\left(\mathrm{S}_{0}, \mathrm{~V}_{0}(\cdot), \mathrm{E}_{0}(\cdot), \mathrm{I}_{0}, \mathrm{R}_{0}(\cdot)\right) \in \partial \mathrm{Y}$, then we have $\left(\mathrm{E}_{0}(\cdot), \mathrm{I}_{0}, \mathrm{R}_{0}(\cdot)\right) \in \partial \hat{\mathrm{Y}}$. We consider the following system,

$$
\left\{\begin{array}{l}
\left(\frac{\partial}{\partial t}+\frac{\partial}{\partial a}\right) E(t, a)=-\sigma(a) E(t, a)-\mu E(t, a) \\
\frac{d I(t)}{d t}=\int_{0}^{\infty} \sigma(a) E(t, a) d a-(\mu+k) I(t)+\int_{0}^{\infty} \gamma(b) R(t, b) d b \\
\left(\frac{\partial}{\partial t}+\frac{\partial}{\partial a}\right) R(t, b)=-\gamma(b) R(t, b)-\mu R(t, b) \\
E(t, 0)=\beta S(t) I(t)+\int_{0}^{\infty} h(a) V(t, a) d a I(t) \\
R(t, 0)=k I(t), \\
E(0, a)=E_{0}(a), I(0)=I_{0}, R(0, b)=R_{0}(b)
\end{array}\right.
$$

Since $S(t) \leqslant \frac{\Lambda}{\mu}, \int_{0}^{\infty} V(t, a) d a \leqslant \frac{\Lambda}{\mu}$ as $t$ tend to the infinity, it follows that

$$
E(t, a) \leqslant \breve{E}(t, a), \quad I(t) \leqslant \breve{I}(t), \quad R(t, b) \leqslant \breve{R}(t, b),
$$

where $\breve{E}(t, a), \breve{I}(t), \breve{R}(t, b)$ satisfy,

$$
\left\{\begin{array}{l}
\left(\frac{\partial}{\partial t}+\frac{\partial}{\partial a}\right) \breve{E}(t, a)=-\sigma(a) \breve{E}(t, a)-\mu \breve{E}(t, a) \\
\frac{d \breve{I}(t)}{d t}=\int_{0}^{\infty} \sigma(a) \breve{E}(t, a) d a-(\mu+k) \breve{I}(t)+\int_{0}^{\infty} \gamma(b) \breve{R}(t, b) d b \\
\left(\frac{\partial}{\partial t}+\frac{\partial}{\partial a}\right) \check{R}(t, b)=-\gamma(b) \breve{R}(t, b)-\mu \breve{R}(t, b) \\
\breve{E}(t, 0)=(\beta+\bar{h}) \frac{\Lambda}{\mu} \breve{I}(t), R(t, 0)=k \breve{I}(t) \\
\breve{E}(0, a)=E_{0}(a), \breve{I}(0)=I_{0}, \breve{R}(0, b)=R_{0}(b) .
\end{array}\right.
$$


Solving $\check{E}(t, a), \check{R}(t, b)$ of (4.2) yields

$$
\breve{E}(t, a):= \begin{cases}\breve{E}(t-a, 0) e^{-\int_{0}^{a}\{\sigma(\tau)+\mu\} d \tau}=(\beta+\bar{h}) \frac{\Lambda}{\mu} \breve{I}(t-a) \Gamma(a), & t>a \geqslant 0, \\ \breve{E}_{0}(a-t) e^{-\int_{a-t}^{a}\{\sigma(\tau)+\mu\} d \tau}=E_{0}(a-t) \frac{\Gamma(a)}{\Gamma(a-t)}, & a \geqslant t \geqslant 0,\end{cases}
$$

and

$$
\breve{R}(t, b):= \begin{cases}\breve{R}(t-b, 0) e^{-\int_{0}^{b}\{\gamma(\tau)+\mu\} d \tau}=k \breve{I}(t-b) \Upsilon(b), & t>b \geqslant 0, \\ \breve{R}_{0}(b-t) e^{-\int_{b-t}^{b}\{\gamma(\tau)+\mu\} d \tau}=R_{0}(b-t) \frac{\gamma(b)}{\gamma(b-t)}, & b \geqslant t \geqslant 0 .\end{cases}
$$

Substituting (4.3) and (4.4) into the second equation of (4.2) gives,

$$
\frac{d}{d t} \breve{I}(t)=(\beta+\bar{h}) \frac{\wedge}{\mu} \int_{0}^{t} \sigma(a) \Gamma(a) \breve{I}(t-a) d a-(\mu+k) \breve{I}(t)+k \int_{0}^{t} \gamma(b) \Upsilon(b) \breve{I}(t-b) d b+Q(t),
$$

where

$$
Q(t)=\int_{t}^{\infty} \sigma(a) E_{0}(a-t) \frac{\Gamma(a)}{\Gamma(a-t)} d a+\int_{t}^{\infty} \gamma(b) R_{0}(b-t) \frac{\Upsilon(b)}{\gamma(b-t)} d b
$$

It follows from $\left(E_{0}(\cdot), I_{0}, R_{0}(\cdot)\right) \in \partial \hat{Y}$ that $Q(t) \equiv 0$ holds true for $t \geqslant 0$. Further we have

$$
\left\{\begin{array}{l}
\frac{d}{d t} \breve{I}(t)=(\beta+\bar{h}) \frac{\Lambda}{\mu} \int_{0}^{t} \sigma(a) \Gamma(a) \breve{I}(t-a) d a-(\mu+k) \breve{I}(t)+k \int_{0}^{t} \gamma(b) \gamma(b) \breve{I}(t-b) d b \\
\breve{I}(0)=0
\end{array}\right.
$$

has a unique solution $\breve{I}(t)=0$. By (4.3) and (4.4), we have $\breve{E}(t, a)=0, \breve{R}(t, b)=0$ for $0 \leqslant a, b \leqslant t$.

Moreover, for $a, b \geqslant t$, it is easy to see that

$$
\|\breve{E}(t, a)\|_{L^{1}}=\left\|E_{0}(a-t) \frac{\Gamma(a)}{\Gamma(a-t)}\right\|_{L^{1}} \leqslant\left\|E_{0}\right\|_{L^{1}} e^{-\left(\mu_{0}+\mu\right) t},
$$

and

$$
\|\breve{R}(t, b)\|_{L^{1}}=\left\|R_{0}(b-t) \frac{\Upsilon(b)}{\Upsilon(b-t)}\right\|_{L^{1}} \leqslant\left\|R_{0}\right\|_{L^{1}} e^{-\left(\mu_{0}+\mu\right) t} .
$$

It follows that $\lim _{t \rightarrow \infty} \breve{E}(t, a)=0, \lim _{t \rightarrow \infty} \breve{R}(t, b)=0$. Thus, from (4.1), $\lim _{t \rightarrow \infty} E(t, a)=0, \lim _{t \rightarrow \infty} I(t)=$ 0 , and $\lim _{t \rightarrow \infty} R(t, b)=0$. This completes the proof.

To proceed, we adopted the method in Hale and Waltman [9] to prove the uniform persistence of system (1.4). We introduce the following lemma.

Lemma 4.2 ([1]). Consider the following scalar Volterra integro-differential equations:

$$
\frac{d J(t)}{d t}=\int_{0}^{\infty} q(\tau) J(t-\tau) d \tau-\kappa J(t), \quad \text { for } \int_{0}^{\infty} q(\tau) d \tau>\kappa>0,
$$

where $\mathrm{q}(\cdot) \in \mathrm{L}_{+}^{1}, \mathrm{~J}(0)>0$. Then there is a unique solution $\mathrm{J}(\mathrm{t})$ that is unbounded.

According to [9, Theorem 5.2], one only needs to verify that there are $T>0$ and $\varepsilon>0$, such that

$$
\lim _{t \rightarrow \infty} \inf \|\Phi(t)\| x \geqslant \varepsilon, \quad \text { for } t>T, \quad x \in Y .
$$

To this end, we define $W^{s}\left(E^{0}\right)=\left\{x \in Y: \lim _{t \rightarrow \infty} \Phi(t, x)=E^{0}\right\}$. It suffices to examine that

$$
W^{s}\left(E^{0}\right) \cap Y=\emptyset \text {. }
$$

By using the same method as in [11, Theorem 5.4], we are able to show the following theorem about the uniform persistence of (1.4). 
Theorem 4.3. Suppose that $\mathfrak{R}_{0}>1$. The semi-flow $\Phi(\mathrm{t})_{\mathrm{t} \geqslant 0}$ is uniformly persistent with respect to $(\mathrm{Y}, \partial \mathrm{Y})$, that is, there exists a constant $\varepsilon>0$ which does not depend on initial data, such that $\lim _{\inf _{\mathrm{t}} \rightarrow \infty}\|\Phi(\mathrm{t})\| y \geqslant \varepsilon$.

Uniform persistence and existence of global attractor $A_{0}$ for $\Phi(t)_{t \geqslant 0}$ now follows immediately from [3, Theorem 3.7].

Theorem 4.4. The semi-flow $\Phi(t)_{t \geqslant 0}$ admits a global attractor $A_{0}$ in $y$, which attracts any bounded subset of $y$.

\section{Stability analysis}

\subsection{Global stability of the disease-free equilibrium}

In fact, we have established the global asymptotic stability of disease-free equilibrium of system (1.4) in Theorem 4.1 when $\mathfrak{R}_{0}>1$. To highlight the role of Lyapunov function techniques in establishing the stability of equilibria, we give the proof again by Lyapunov function techniques. Before going into details, denote

$$
\mathcal{G}(z)=z-1-\ln z, \quad \forall z>0 .
$$

Obviously, $\mathcal{G}: \mathbb{R}^{+} \rightarrow \mathbb{R}^{+}$attains its strict global minimum at $z=1$ and $\mathcal{G}(z) \geqslant \mathcal{G}(1)=0$.

Define two key functions as

$$
\omega_{1}(a)=\int_{a}^{+\infty} \sigma(s) e^{-\int_{a}^{s} \varepsilon(\tau) d \tau} d s, \quad \omega_{2}(b)=\int_{a}^{+\infty} \gamma(s) e^{-\int_{b}^{s} \eta(\tau) d \tau} d s,
$$

it follows that $\omega_{1}(a), \omega_{2}(b)>0$ for $a, b \geqslant 0$ and $\omega_{i}(0)=\theta_{i}$ for $i=1,2$. The derivative of $\omega_{1}(a), \omega_{2}(b)$ satisfy

$$
\frac{d}{d a} \omega_{1}(a)=\omega_{1}(a) \varepsilon(a)-\sigma(a), \quad \frac{d}{d a} \omega_{2}(b)=\omega_{2}(b) \eta(b)-\gamma(b) .
$$

Theorem 5.1. Consider system (1.4) with $\mathfrak{R}_{0}$ defined by (2.5). The disease-free equilibrium of system (1.4) is globally asymptotically stable if $\Re_{0} \leqslant 1$ and it is unstable if $\Re_{0}>1$.

Proof. Consider a Lyapunov functional

$$
\mathrm{L}_{\mathrm{DFE}}(\mathrm{t})=\mathrm{L}_{1}(\mathrm{t})+\mathrm{L}_{2}(\mathrm{t})+\mathrm{L}_{3}(\mathrm{t})+\mathrm{I}(\mathrm{t})+\mathrm{L}_{4}(\mathrm{t}),
$$

where

$$
\left\{\begin{array}{l}
\mathrm{L}_{1}(\mathrm{t})=\theta_{1} S^{0} \mathcal{G}\left(\frac{\mathrm{S}}{\mathrm{S}^{0}}\right), \\
\mathrm{L}_{2}(\mathrm{t})=\theta_{1} \int_{0}^{\infty} \mathrm{V}^{0}(\mathrm{a}) \mathcal{G}\left(\frac{\mathrm{V}(\mathrm{t}, \mathrm{a})}{\mathrm{V}^{0}(\mathrm{a})}\right) d a, \\
\mathrm{~L}_{3}(\mathrm{t})=\int_{0}^{\infty} \omega_{1}(\mathrm{a}) \mathrm{E}(\mathrm{t}, \mathrm{a}) \mathrm{da}, \\
\mathrm{L}_{4}(\mathrm{t})=\int_{0}^{\infty} \omega_{2}(\mathrm{~b}) \mathrm{R}(\mathrm{t}, \mathrm{b}) \mathrm{d} b .
\end{array}\right.
$$

Note that $\mu S^{0}=(1-p) \Lambda+\int_{0}^{\infty} \alpha(a) V^{0}(a) d a$. Calculating the derivative of $L_{1}(t)$ along with the solutions of system (1.4) gives

$$
\begin{aligned}
\frac{d L_{1}(t)}{d t}= & \theta_{1}\left(1-\frac{S^{0}}{S(t)}\right)\left[(1-p) \wedge\left(1-\frac{S(t)}{S^{0}}\right)-\beta S(t) I(t)+\int_{0}^{\infty} \alpha(a) V^{0}(a)\left(\frac{V(t, a)}{V^{0}(a)}-\frac{S(t)}{S^{0}}\right) d a\right] \\
= & \theta_{1}(1-p) \wedge\left(2-\frac{S^{0}}{S(t)}-\frac{S(t)}{S^{0}}\right)+\theta_{1} \int_{0}^{\infty} \alpha(a) V^{0}(a)\left(\frac{V(t, a)}{V^{0}(a)}-\frac{S(t)}{S^{0}}-\frac{S^{0} V(t, a)}{S V^{0}(a)}+1\right) d a \\
& +\theta_{1} \beta S^{0} I(t)-\theta_{1} \beta S(t) I(t) .
\end{aligned}
$$


The derivative of $L_{2}(t)$ satisfies,

$$
\begin{aligned}
\frac{d L_{2}(t)}{d t} & =\theta_{1} \int_{0}^{\infty} V^{0}(a) \frac{\partial}{\partial t} \mathcal{G}\left(\frac{V(t, a)}{V^{0}(a)}\right) d a \\
& =-\theta_{1} \int_{0}^{\infty} V^{0}(a)\left(\frac{1}{V^{0}(a)}-\frac{1}{V(t, a)}\right)\left((\mu+\alpha(a)+h(a) I(t)) V(t, a)+\frac{\partial V(t, a)}{\partial a}\right) d a \\
& =-\theta_{1} \int_{0}^{\infty} V^{0}(a)\left(\frac{V(t, a)}{V^{0}(a)}-1\right)\left(\frac{V_{a}(t, a)}{V(t, a)}+\mu+\alpha(a)+h(a) I(t)\right) d a,
\end{aligned}
$$

where $V_{a}(t, a)$ denotes $\frac{\partial V(t, a)}{\partial a}$. Recall that $\frac{d V^{0}(a)}{d a}=-(\mu+\alpha(a)) V^{0}(a)$, it follows that

$$
\frac{\partial}{\partial a} \mathcal{G}\left(\frac{V(t, a)}{V^{0}(a)}\right)=\left(\frac{V(t, a)}{V^{0}(a)}-1\right)\left(\frac{V_{a}(t, a)}{V(t, a)}+\mu+\alpha(a)\right)
$$

Using integration by parts, and $\mathrm{V}^{0}(0)=\mathrm{p} \wedge, \mathrm{V}(\mathrm{t}, 0)=\mathrm{p} \wedge$, we have

$$
\begin{aligned}
\frac{d L_{2}(t)}{d t}= & -\theta_{1} \int_{0}^{\infty} V^{0}(a) \frac{\partial}{\partial a} \mathcal{G}\left(\frac{V(t, a)}{V^{0}(a)}\right) d a-\theta_{1} \int_{0}^{\infty} V^{0}(a)\left(\frac{V(t, a)}{V^{0}(a)}-1\right) h(a) d a I(t) \\
= & -\left.\theta_{1} V^{0}(a) \mathcal{G}\left(\frac{V(t, a)}{V^{0}(a)}\right)\right|_{a=\infty}-\theta_{1} \int_{0}^{\infty} V^{0}(a)(\mu+\alpha(a)) \mathcal{G}\left(\frac{V(t, a)}{V^{0}(a)}\right) d a \\
& -\theta_{1} \int_{0}^{\infty} h(a) V(t, a) \operatorname{daI}(t)+\theta_{1} \int_{0}^{\infty} h(a) V^{0}(a) d a I(t) .
\end{aligned}
$$

The derivative of $L_{3}(t)$ satisfies

$$
\begin{aligned}
\frac{d L_{3}(t)}{d t} & =\omega_{1}(0) E(t, 0)-\int_{0}^{\infty} \sigma(a) E(t, a) d a \\
& =\theta_{1}\left(\beta S(t) I(t)+\int_{0}^{\infty} h(a) V(t, a) d a I(t)\right)-\int_{0}^{\infty} \sigma(a) E(t, a) d a
\end{aligned}
$$

Here we use the fact that $\omega_{1}(0)=\theta_{1}$ and $E(t, 0)=\beta S I+\int_{0}^{\infty} h(a) V(t, a) d a I(t)$.

Similarly, by using $\omega_{2}(0)=\theta_{2}$ and $R(t, 0)=k I(t)$, the derivative of $L_{4}(t)$ becomes

$$
\frac{d L_{4}(t)}{d t}=\theta_{2} k I(t)-\int_{0}^{\infty} \gamma(b) R(t, b) d b .
$$

It follows from (5.1), (5.2), (5.3), (5.4) that

$$
\frac{d L_{D F E}(t)}{d t}=-\left.\theta_{1} V^{0}(a) \mathcal{G}\left(\frac{V(t, a)}{V^{0}(a)}\right)\right|_{a=\infty}-\theta_{1} \mu \int_{0}^{\infty} V^{0}(a) \mathcal{G}\left(\frac{V(t, a)}{V^{0}(a)}\right) d a+\bigoplus_{1}+\bigoplus_{2}+\bigoplus_{3}
$$

where

$$
\begin{gathered}
\bigoplus_{1}=\theta_{1}(1-p) \wedge\left(2-\frac{S^{0}}{S(t)}-\frac{S(t)}{S^{0}}\right) \\
=-\theta_{1}(1-p) \wedge\left[\mathcal{G}\left(\frac{S^{0}}{S(t)}\right)+\mathcal{G}\left(\frac{S(t)}{S^{0}}\right)\right] \\
\bigoplus_{2}=\theta_{1} \int_{0}^{\infty} \alpha(a) V^{0}(a)\left(-\frac{S(t)}{S^{0}}-\frac{S^{0} V(t, a)}{S(t) V^{0}(a)}+2+\ln \frac{V(t, a)}{V^{0}(a)}\right) d a
\end{gathered}
$$




$$
\begin{aligned}
& =\theta_{1} \int_{0}^{\infty} \alpha(a) V^{0}(a)\left(-\frac{S(t)}{S^{0}}-\frac{S^{0} V(t, a)}{S(t) V^{0}(a)}+2+\ln \frac{S(t)}{S^{0}}+\ln \frac{S^{0} V(t, a)}{S(t) V^{0}(a)}\right) d a \\
& =-\theta_{1} \int_{0}^{\infty} \alpha(a) V^{0}(a)\left[\mathcal{G}\left(\frac{S(t)}{S^{0}}\right)+\mathcal{G}\left(\frac{S^{0} V(t, a)}{S(t) V^{0}(a)}\right)\right] d a
\end{aligned}
$$

and

$$
\begin{aligned}
\bigoplus_{3} & =\theta_{1} \beta S^{0} I(t)+\theta_{1} \int_{0}^{\infty} h(a) V^{0}(a) I(t) d a+\theta_{2} k I(t)-(\mu+k) I(t) \\
& =\left[(\mu+k)-k \theta_{2}\right]\left(\Re_{0}-1\right) I(t) .
\end{aligned}
$$

Consequently, we have that

$$
\begin{aligned}
\frac{d L_{D F E}(t)}{d t}= & -\left.\theta_{1} V^{0}(a) \mathcal{G}\left(\frac{V(t, a)}{V^{0}(a)}\right)\right|_{a=\infty}-\theta_{1} \mu \int_{0}^{\infty} V^{0}(a) \mathcal{G}\left(\frac{V(t, a)}{V^{0}(a)}\right) d a \\
& -\theta_{1}(1-p) \wedge\left[\mathcal{G}\left(\frac{S^{0}}{S(t)}\right)+\mathcal{G}\left(\frac{S(t)}{S^{0}}\right)\right] \\
& -\theta_{1} \int_{0}^{\infty} \alpha(a) V^{0}(a)\left[\mathcal{G}\left(\frac{S(t)}{S^{0}}\right)+\mathcal{G}\left(\frac{S^{0} V(t, a)}{S(t) V^{0}(a)}\right)\right] d a+\left[(\mu+k)-k \theta_{2}\right]\left(\Re_{0}-1\right) I(t) .
\end{aligned}
$$

Therefore, $\mathfrak{R}_{0} \leqslant 1$ ensures that $\frac{d L_{D F E}(t)}{d t} \leqslant 0$ holds. Furthermore, the strict equality holds only if $S(t)=S^{0}$, $V(t, a)=V^{0}(a)$ and $I(t)=0$, simultaneously. By the Lyapunov-Lasalle invariance principle, the diseasefree equilibrium $E^{0}$ is globally asymptotically stable when $\mathfrak{R}_{0} \leqslant 1$.

\subsection{Global stability of endemic equilibrium}

In this subsection, a sharp criterion for the global asymptotic stability of the endemic equilibrium of (1.4) is established. When $\mathfrak{R}_{0}>1$, we know that $E^{0}$ becomes unstable and (1.4) also has an endemic equilibrium $E^{*}$ in addition to $E^{0}$. In the section, we discuss the stability of this endemic equilibrium.

Theorem 5.2. Consider system (1.4). If $\mathfrak{R}_{0}>1$, endemic equilibrium $\mathrm{E}^{*}$ is globally asymptotically stable.

Proof. Define a Lyapunov functional as

$$
L_{E E}(t)=W_{1}(t)+W_{2}(t)+W_{3}(t)+W_{4}(t)+W_{5}(t)
$$

where

$$
\left\{\begin{array}{l}
W_{1}(t)=\theta_{1} S^{*} \mathcal{G}\left(\frac{S(t)}{S^{*}}\right), \\
W_{2}(t)=\theta_{1} \int_{0}^{\infty} V^{*}(a) \mathcal{G}\left(\frac{V(t, a)}{V^{*}(a)}\right) d a, \\
W_{3}(t)=\int_{0}^{\infty} \omega_{1}(a) E^{*}(a) \mathcal{G}\left(\frac{E(t, a)}{E^{*}(a)}\right) d a, \\
W_{4}(t)=I^{*} \mathcal{G}\left(\frac{I(t)}{I^{*}}\right), \\
W_{5}(t)=\int_{0}^{\infty} \omega_{2}(b) R^{*}(b) \mathcal{G}\left(\frac{R(t, b)}{R^{*}(b)}\right) d b .
\end{array}\right.
$$

Calculating the derivative of $W_{1}(t)$ by $\mu S^{*}=(1-p) \Lambda-\beta S^{*} I^{*}+\int_{0}^{\infty} \alpha(a) V^{*}(a)$ da gives

$$
\begin{aligned}
\frac{d W_{1}(t)}{d t} & =\theta_{1}\left(1-\frac{S^{*}}{S(t)}\right)\left((1-p) \Lambda-\mu S(t)-\beta S(t) I(t)+\int_{0}^{\infty} \alpha(a) V(t, a) d a\right) \\
& =\theta_{1}(1-p) \wedge\left(2-\frac{S^{*}}{S(t)}-\frac{S(t)}{S^{*}}\right)+\theta_{1} \beta S^{*} I^{*}\left(\frac{S(t)}{S^{*}}-\frac{S(t) I(t)}{S^{*} I^{*}}-1+\frac{I(t)}{I^{*}}\right)
\end{aligned}
$$




$$
+\theta_{1} \int_{0}^{\infty} \alpha(a) V^{*}(a)\left(\frac{V(t, a)}{V^{*}(a)}-\frac{S(t)}{S^{*}}-\frac{S^{*} V(t, a)}{S(t) V^{*}(a)}+1\right) d a
$$

The derivative of $W_{2}(t)$ along with the solutions of system (1.4) satisfies

$$
\begin{aligned}
\frac{d W_{2}(t)}{d t} & =\theta_{1} \int_{0}^{\infty} V^{*}(a)\left(\frac{1}{V^{*}(a)}-\frac{1}{V(t, a)}\right) \frac{\partial}{\partial t} V(t, a) d a \\
& =-\theta_{1} \int_{0}^{\infty} V^{*}(a)\left(\frac{V(t, a)}{V^{*}(a)}-1\right)\left[\frac{V_{a}(t, a)}{V(t, a)}+n^{*}(a)+h(a) I^{*}\left(\frac{I(t)}{I^{*}}-1\right)\right] d a
\end{aligned}
$$

Note that $\frac{d V^{*}(a)}{d a}=-n^{*}(a) V^{*}(a)$, it follows that

$$
\frac{\partial}{\partial a} \mathcal{G}\left(\frac{V(t, a)}{V^{*}(a)}\right)=\left(\frac{V(t, a)}{V^{*}(a)}-1\right)\left(\frac{V_{a}(t, a)}{V(t, a)}+n^{*}(a)\right)
$$

and

$$
\mathcal{G}\left(\frac{\mathrm{V}(\mathrm{t}, 0)}{\mathrm{V}^{*}(0)}\right)=\mathcal{G}(1)=0
$$

Thus, (5.6) becomes

$$
\begin{aligned}
\frac{d W_{2}(t)}{d t}= & -\theta_{1} \int_{0}^{\infty} V^{*}(a) \frac{\partial}{\partial a} \mathcal{G}\left(\frac{V(t, a)}{V^{*}(a)}\right) d a-\theta_{1} \int_{0}^{\infty} V^{*}(a) h(a) I^{*}\left(\frac{V(t, a)}{V^{*}(a)}-1\right)\left(\frac{I(t)}{I^{*}}-1\right) d a \\
= & -\left.\theta_{1} V^{*}(a) \mathcal{G}\left(\frac{V(t, a)}{V^{*}(a)}\right)\right|_{a=\infty}-\theta_{1} \int_{0}^{\infty} V^{*}(a)\left[h(a) I^{*}+\alpha(a)+\mu\right] \mathcal{G}\left(\frac{V(t, a)}{V^{*}(a)}\right) d a \\
& -\theta_{1} \int_{0}^{\infty} V^{*}(a) h(a) I^{*}\left(\frac{V(t, a) I(t)}{V^{*}(a) I^{*}}-\frac{V(t, a)}{V^{*}(a)}-\frac{I(t)}{I^{*}}+1\right) d a \\
= & -\left.\theta_{1} V^{*}(a) \mathcal{G}\left(\frac{V(t, a)}{V^{*}(a)}\right)\right|_{a=\infty}-\theta_{1} \int_{0}^{\infty} V^{*}(a)[\alpha(a)+\mu] \mathcal{G}\left(\frac{V(t, a)}{V^{*}(a)}\right) d a \\
& -\theta_{1} \int_{0}^{\infty} V^{*}(a) h(a) I^{*}\left(\frac{V(t, a) I(t)}{V^{*}(a) I^{*}}-\frac{I(t)}{I^{*}}-\ln \frac{V(t, a)}{V^{*}(a)}\right) d a .
\end{aligned}
$$

Similarly, the derivative of $W_{3}(t)$ satisfies

$$
\begin{aligned}
\frac{d W_{3}(t)}{d t} & =-\int_{0}^{\infty} \omega_{1}(a)\left(1-\frac{E^{*}(a)}{E(t, a)}\right)\left(\frac{\partial}{\partial a} E(t, a)+\varepsilon(a) E(t, a)\right) d a \\
& =-\int_{0}^{\infty} \omega_{1}(a) E^{*}(a)\left(\frac{E(t, a)}{E^{*}(a)}-1\right)\left(\frac{E_{a}(t, a)}{E(t, a)}+\varepsilon(a)\right) d a
\end{aligned}
$$

where $E_{a}(t, a)$ denotes $\frac{\partial E(t, a)}{\partial a}$. Recall that

$$
\frac{\partial}{\partial a} \mathcal{G}\left(\frac{E(t, a)}{E^{*}(a)}\right)=\left(\frac{E(t, a)}{E^{*}(a)}-1\right)\left(\frac{E_{a}(t, a)}{E(t, a)}+\varepsilon(a)\right)
$$

and

$$
\frac{d \omega_{1}(a)}{d a}=\omega_{1}(a) \varepsilon(a)-\sigma(a), \quad \frac{d E^{*}(a)}{d a}=-\varepsilon(a) E^{*}(a) .
$$

Thus, (5.8) becomes

$$
\frac{d W_{3}(t)}{d t}=-\int_{0}^{\infty} \omega_{1}(a) E^{*}(a) \frac{\partial}{\partial a} \mathcal{G}\left(\frac{E(t, a)}{E^{*}(a)}\right) d a
$$




$$
\begin{aligned}
= & -\left.\omega_{1}(a) E^{*}(a) \mathcal{G}\left(\frac{E(t, a)}{E^{*}(a)}\right)\right|_{a=0} ^{a=\infty}+\int_{0}^{\infty} E^{*}(a) \mathcal{G}\left(\frac{E(t, a)}{E^{*}(a)}\right) \frac{d \omega_{1}(a)}{d a} d a \\
& +\int_{0}^{\infty} \omega_{1}(a) \mathcal{G}\left(\frac{E(t, a)}{E^{*}(a)}\right) \frac{d E^{*}(a)}{d a} d a \\
= & \theta_{1} E^{*}(0) \mathcal{G}\left(\frac{E(t, 0)}{E^{*}(0)}\right)-\int_{0}^{\infty} \sigma(a) E^{*}(a) \mathcal{G}\left(\frac{E(t, a)}{E^{*}(a)}\right) d a \\
= & \theta_{1} E(t, 0)-\theta_{1} E^{*}(0)\left(1+\ln \frac{E(t, 0)}{E^{*}(0)}\right)-\int_{0}^{\infty} \sigma(a) E^{*}(a) \mathcal{G}\left(\frac{E(t, a)}{E^{*}(a)}\right) d a .
\end{aligned}
$$

Note that $\omega_{1}(0)=\theta_{1}, E^{*}(0)=\beta S^{*} I^{*}+\int_{0}^{\infty} h(a) E^{*}(a) d a I^{*}, E(t, 0)=\beta S(t) I(t)+\int_{0}^{\infty} h(a) V(t, a) d a I(t) . B y$ a zero trick, we calculate the derivative of $W_{4}(t)$ and $W_{5}(t)$ as follows,

$$
\begin{aligned}
\frac{d W_{4}(t)}{d t}= & \left(1-\frac{I^{*}}{I(t)}\right)\left(\int_{0}^{\infty} \sigma(a) E(t, a) d a+\int_{0}^{\infty} \gamma(b) R(t, b) d b-\left(\mu+\delta_{i}+k\right) I(t)\right) \\
= & \left(1-\frac{I^{*}}{I(t)}\right)\left[\int_{0}^{\infty} \sigma(a) E^{*}(a)\left(\frac{E(t, a)}{E^{*}(a)}-\frac{I(t)}{I^{*}}\right) d a+\int_{0}^{\infty} \gamma(b) R^{*}(b)\left(\frac{R(t, b)}{R^{*}(b)}-\frac{I(t)}{I^{*}}\right) d b\right] \\
= & \int_{0}^{\infty} \sigma(a) E^{*}(a)\left(1-\frac{I(t)}{I^{*}}-\frac{I^{*} E(t, a)}{I E^{*}(a)}+\frac{E(t, a)}{E^{*}(a)}\right) d a \\
& +\int_{0}^{\infty} \gamma(b) R^{*}(b)\left(1-\frac{I^{(}(t)}{I^{*}}-\frac{I^{*} R(t, b)}{I R^{*}(b)}+\frac{R(t, b)}{R^{*}(b)}\right) d b,
\end{aligned}
$$

and

$$
\frac{d W_{5}(t)}{d t}=\omega_{2}(0) R^{*}(0) \mathcal{G}\left(\frac{R(t, 0)}{R^{*}(0)}\right)-\int_{0}^{\infty} \gamma(b) R^{*}(b) \mathcal{G}\left(\frac{R(t, b)}{R^{*}(b)}\right) d b .
$$

Note that $\omega_{2}(0)=\theta_{2}, R^{*}(0)=k I^{*}, R(t, 0)=k I$, Thus, (5.11) becomes

$$
\frac{d W_{5}(t)}{d t}=\theta_{2} k^{*} \mathcal{G}\left(\frac{I(t)}{I^{*}}\right)+\int_{0}^{\infty} \gamma(b) R^{*}(b)\left(1-\frac{R(t, b)}{R^{*}(b)}+\ln \frac{R(t, b)}{R^{*}(b)}\right) d b .
$$

Recall that $E(t, 0)=\beta S I+\int_{0}^{\infty} h(a) V(t, a) d a I, E^{*}(0)=\beta S^{*} I^{*}+\int_{0}^{\infty} h(a) V^{*}(a) d a I^{*}$. It follows from (5.5), (5.6), (5.7), (5.8), (5.9), (5.10), (5.11), (5.12), we can get that

$$
\frac{d L_{E E}(t)}{d t}=-\left.\theta_{1} V^{*}(a) \mathcal{G}\left(\frac{V(t, a)}{V^{*}(a)}\right)\right|_{a=\infty}-\theta_{1} \mu \int_{0}^{\infty} V^{*}(a) \mathcal{G}\left(\frac{V(t, a)}{V^{*}(a)}\right) d a+\sum_{i=1}^{6} \bigotimes_{i},
$$

where

$$
\left\{\begin{array}{l}
\bigotimes=-\theta_{1}(1-p) \wedge\left(\frac{S(t)}{S^{*}}+\frac{S^{*}}{S(t)}-2\right), \\
\bigotimes=\theta_{1} \beta S^{*} I^{*}\left(\frac{S(t)}{S^{*}}+\frac{I(t)}{I^{*}}-2-\ln \frac{E(t, 0)}{E^{*}(0)}\right), \\
\bigotimes=\theta_{1} \int_{0}^{\infty} \alpha(a) V^{*}(a)\left(-\frac{S(t)}{S^{*}}-\frac{S^{*} V(t, a)}{S(t) V^{*}(a)}+2+\ln \frac{V(t, a)}{V^{*}(a)}\right) d a, \\
\bigotimes=\theta_{1} \int_{0}^{\infty} h(a) V^{*}(a) I^{*}\left(\frac{I(t)}{I^{*}}+\ln \frac{V(t, a)}{V^{*}(a)}-1-\ln \frac{E(t, 0)}{E^{*}(0)}\right) d a, \\
\bigotimes=-\int_{0}^{\infty} \sigma(a) E^{*}(a) \mathcal{G}\left(\frac{E(t, a)}{E^{*}(a)}\right) d a+\int_{0}^{\infty} \sigma(a) E^{*}(a)\left(1-\frac{I(t)}{I^{*}}-\frac{I^{*} E(t, a)}{I E *(a)}+\frac{E(t, a)}{E^{*}(a)}\right) d a, \\
\bigotimes=\int_{0}^{\infty} \gamma(b) R^{*}(b)\left(-\frac{I(t)}{I^{*}}-\frac{I^{*} R(t, b)}{I(t) R^{*}(b)}+2+\ln \frac{R(t, b)}{R^{*}(b)}\right) d b+\theta_{2} I^{*} \mathcal{G}\left(\frac{I(t)}{I^{*}}\right) .
\end{array}\right.
$$


Obviously, $\otimes_{1}$ and $\bigotimes_{3}$ can be rewritten as

$$
\bigotimes_{1}=-\theta_{1}(1-p) \wedge\left[\mathcal{G}\left(\frac{S(t)}{S^{*}}\right)+\mathcal{G}\left(\frac{S^{*}}{S(t)}\right)\right]
$$

and

$$
\bigotimes_{3}=-\theta_{1} \int_{0}^{\infty} \alpha(a) V^{*}(a)\left[\mathcal{G}\left(\frac{S(t)}{S^{*}}\right)+\mathcal{G}\left(\frac{S^{*} V(t, a)}{S(t) V^{*}(a)}\right)\right] d a
$$

By using $E^{*}(a)=\left(\beta S^{*} I^{*}+\int_{0}^{\infty} h(a) V^{*}(a) d a I^{*}\right) \Gamma(a)$, we have

$$
\begin{aligned}
\underset{5}{\bigotimes} & =\int_{0}^{\infty} \sigma(a) E^{*}(a)\left(-\frac{I(t)}{I^{*}}-\frac{I^{*} E(t, a)}{I(t) E^{*}(a)}+2+\ln \frac{E(t, a)}{E^{*}(a)}\right) d a \\
& =-\int_{0}^{\infty} \sigma(a) E^{*}(a)\left[\mathcal{G}\left(\frac{I(t)}{I^{*}}\right)+\mathcal{G}\left(\frac{I^{*} E(t, a)}{I(t) E^{*}(a)}\right)\right] d a
\end{aligned}
$$

By using $\theta_{2} \mathrm{kI}^{*}=\int_{0}^{\infty} \gamma(\mathrm{b}) \Upsilon(\mathrm{b}) \mathrm{kI}^{*} \mathrm{db}=\int_{0}^{\infty} \gamma(\mathrm{b}) \mathrm{R}^{*}(\mathrm{~b}) \mathrm{db}$, we have

$$
\begin{aligned}
\bigotimes_{6} & =-\int_{0}^{\infty} \gamma(b) R^{*}(b) \mathcal{G}\left(\frac{I(t)}{I^{*}}\right) d b-\int_{0}^{\infty} \gamma(b) R^{*}(b) \mathcal{G}\left(\frac{I^{*} R(t, b)}{I(t) R^{*}(b)}\right) d b+\theta_{2} k I^{*} \mathcal{G}\left(\frac{I(t)}{I^{*}}\right) \\
& =-\int_{0}^{\infty} \gamma(b) R^{*}(b) \mathcal{G}\left(\frac{I^{*} R(t, b)}{I(t) R^{*}(b)}\right) d b .
\end{aligned}
$$

By using the fact that $-\ln \frac{E(t, 0)}{E^{*}(0)}=-\ln \frac{S(t)}{S^{*}}-\ln \frac{I(t)}{I^{*}}+\ln \frac{S(t) I(t) E^{*}(0)}{S^{*} I^{*} E(t, 0)}$, and

$$
\ln \frac{V(t, a)}{V^{*}(a)}-\ln \frac{E(t, 0)}{E^{*}(0)}=\ln \frac{V(t, a) I(t) E^{*}(0)}{V^{*}(a) I^{*} E(t, 0)}-\ln \frac{I(t)}{I^{*}}
$$

which lead to

$$
\bigotimes_{2}=\theta_{1} \beta S^{*} I^{*}\left[\mathcal{G}\left(\frac{S}{S^{*}}\right)+\mathcal{G}\left(\frac{I(t)}{I^{*}}\right)-\mathcal{G}\left(\frac{S(t) I(t) E^{*}(0)}{S^{*} I^{*} E(t, 0)}\right)-1+\frac{S(t) I(t) E^{*}(0)}{S^{*} I^{*} E(t, 0)}\right],
$$

and

$$
\bigotimes_{4}=\theta_{1} \int_{0}^{\infty} h(a) V^{*}(a) I^{*}\left[\mathcal{G}\left(\frac{I(t)}{I^{*}}\right)-\mathcal{G}\left(\frac{V(t, a) I(t) E^{*}(0)}{V^{*}(a) I^{*} E(t, 0)}\right)-1+\frac{V(t, a) I(t) E^{*}(0)}{V^{*}(a) I^{*} E(t, 0)}\right] d a
$$

Further, it is easy to check that

$$
\beta S^{*} I^{*}\left(-1+\frac{S(t) I(t) E^{*}(0)}{S^{*} I^{*} E(t, 0)}\right)+\int_{0}^{\infty} h(a) V^{*}(a) I^{*}\left(-1+\frac{V(t, a) I(t) E^{*}(0)}{V^{*}(a) I^{*} E(t, 0)}\right) d a=0 .
$$

Consequently, we simplify (5.13) as follows

$$
\begin{aligned}
\frac{d L_{E E}(t)}{d t}= & -\theta_{1}(1-p) \wedge \mathcal{G}\left(\frac{S^{*}}{S(t)}\right)-\theta_{1} \mu S^{*} \mathcal{G}\left(\frac{S(t)}{S^{*}}\right)-\left.\theta_{1} V^{*}(a) \mathcal{G}\left(\frac{V(t, a)}{V^{*}(a)}\right)\right|_{a=\infty} \\
& -\theta_{1} \mu \int_{0}^{\infty} V^{*}(a) \mathcal{G}\left(\frac{V(t, a)}{V^{*}(a)}\right) d a-\theta_{1} \int_{0}^{\infty} \alpha(a) V^{*}(a) \mathcal{G}\left(\frac{S^{*} V(t, a)}{S(t) V^{*}(a)}\right) d a \\
& -\int_{0}^{\infty} \sigma(a) E^{*}(a) \mathcal{G}\left(\frac{I^{*} E(t, a)}{I(t) E^{*}(a)}\right) d a-\int_{0}^{\infty} \gamma(b) R^{*}(b) \mathcal{G}\left(\frac{I^{*} R(t, b)}{I(t) R^{*}(t, b)}\right) d b
\end{aligned}
$$




$$
-\theta_{1} \beta S^{*} I^{*} \mathcal{G}\left(\frac{S(t) I(t) E^{*}(0)}{S^{*} I^{*} E(t, 0)}\right)-\theta_{1} \int_{0}^{\infty} h(a) V^{*}(a) I^{*} \mathcal{G}\left(\frac{V(t, a) I(t) E^{*}(0)}{V^{*}(a) I^{*} E(t, 0)}\right) d a .
$$

Therefore, $\frac{d L_{E E}(t)}{d t} \leqslant 0$ holds. Furthermore, the strict equality holds only if $S^{*}=S(t), V^{*}(a)=V(t, a)$, $E^{*}(a)=E(t, a), R^{*}(b)=R(t, b), R(t, b) I^{*}=R^{*}(b) I(t), E(t, a) I^{*}=E^{*}(a) I(t), V(t, a) S^{*}=V^{*}(a) S(t)$, $S(t) I(t) E^{*}(0)=S^{*} I^{*} E(t, 0)$ and $V(t, a) I(t) E^{*}(0)=V^{*}(a) I^{*} E(t, 0)$ simultaneously. Thus, $\left\{E^{*}\right\} \subset \Omega$ is the largest invariant subset of $\left\{\frac{d L_{E E}(t)}{d t}=0\right\}$, and by the Lyapunov-LaSalle invariance principle, the endemic equilibrium $E^{*}$ is globally asymptotically stable when $\mathfrak{R}_{0}>1$.

\section{Acknowledgment}

The authors would like to thank the editors and the referees for their helpful comments. J. L. Wang was supported by National Natural Science Foundation of China (Nos. 11401182, 11471089), Science and Technology Innovation Team in Higher Education Institutions of Heilongjiang Province (No. 2014TD005), Youth Scientific Foundation of Heilongjiang University (QL201203). H. Q. Sun was supported by Research Foundation of Heilongjiang University (HDJCCX-201618).

\section{References}

[1] C. J. Browne, S. S. Pilyugin, Global analysis of age-structured within-host virus model, Discrete Contin. Dyn. Syst. Ser. B, 18 (2013), 1999-2017. 1, 4.2

[2] Y.-M. Chen, S.-F. Zou, J.-Y. Yang, Global analysis of an SIR epidemic model with infection age and saturated incidence, Nonlinear Anal. Real World Appl., 30 (2016), 16-31. 1

[3] R. D. Demasse, A. Ducrot, An age-structured within-host model for multistrain malaria infections, SIAM J. Appl. Math., 73 (2013), 572-593. 4

[4] X.-C. Duan, S.-L. Yuan, X.-Z. Li, Global stability of an SVIR model with age of vaccination, Appl. Math. Comput., 226 (2014), 528-540. 1

[5] W. J. Edmunds, G. F. Medley, D. J. Nokes, A. J. Hall, H. C. Whittle, The influence of age on the development of the hepatitis B carrier state, Proc. R. Soc. Lond. B Biol. Sci., 253 (1993), 197-201. 1

[6] D. Ganem, A. M. Prince, Hepatitis B virus infection—natural history and clinical consequences, N. Engl. J. Med., 350 (2004), 1118-1129. 1

[7] J. K. Hale, Functional differential equations, Applied Mathematical Sciences, Springer-Verlag New York, New YorkHeidelberg, (1971). 3, 3.2

[8] J. K. Hale, Asymptotic behavior of dissipative systems, Mathematical Surveys and Monographs, American Mathematical Society, Providence, RI, (1988). 3.2

[9] J. K. Hale, P. Waltman, Persistence in infinite-dimensional systems, SIAM J. Math. Anal., 20 (1989), 388-395. 1, 4, 4

[10] M. Iannelli, Mathematical theory of age-structured population dynamics, Appl. Math. Monogr. C.N.R., Giardini Editori e Stampatori in Pisa, (1995). 1, 2.1

[11] L.-L. Liu, J.-L. Wang, X.-N. Liu, Global stability of an SEIR epidemic model with age-dependent latency and relapse, Nonlinear Anal. Real World Appl., 24 (2015), 18-35. 1, 1, 4, 4

[12] P. Magal, Compact attractors for time-periodic age-structured population models, Electron. J. Differential Equations, 2001 (2001), 35 pages. $1,2.1$

[13] P. Magal, C. C. McCluskey, Two-group infection age model including an application to nosocomial infection, SIAM J. Appl. Math., 73 (2013), 1058-1095 1

[14] P. Magal, C. C. McCluskey, G. F. Webb, Lyapunov functional and global asymptotic stability for an infection-age model, Appl. Anal., 89 (2010), 1109-1140. 1

[15] P. Magal, X.-Q. Zhao, Global attractors and steady states for uniformly persistent dynamical systems, SIAM J. Math. Anal., 37 (2005), 251-275. 1

[16] C. C. McCluskey, Global stability for an SEI epidemiological model with continuous age-structure in the exposed and infectious classes, Math. Biosci. Eng., 9 (2012), 819-841. 1, 1

[17] L.-L. Rong, Z.-L. Feng, A. S. Perelson, Mathematical analysis of age-structured HIV-1 dynamics with combination antiretroviral therapy, SIAM J. Appl. Math., 67 (2007), 731-756. 1

[18] H. R. Thieme, Semiflows generated by Lipschitz perturbations of non-densely defined operators, Differential Integral Equations, 3 (1990), 1035-1066. 1, 2.1

[19] H. R. Thieme, Global stability of the endemic equilibrium in infinite dimension: Lyapunov functions and positive operators, J. Differential Equations, 250 (2011), 3772-3801. 3.1 
[20] P. van den Driessche, L. Wang, X.-F. Zou, Modeling diseases with latency and relapse, Math. Biosci. Eng., 4 (2007), 205-219. 1

[21] P. van den Driessche, X.-F. Zou, Modeling relapse in infectious diseases, Math. Biosci., 207 (2007), 89-103. 1

[22] J. A. Walker, Dynamical systems and evolution equations, Theory and applications, Mathematical Concepts and Methods in Science and Engineering, Plenum Press, New York-London, (1980). 3

[23] J.-L. Wang, R. Zhang, T. Kuniya, Global dynamics for a class of age-infection HIV models with nonlinear infection rate, J. Math. Anal. Appl., 432 (2015), 289-313. 1

[24] J.-L. Wang, R. Zhang, T. Kuniya, The stability analysis of an SVEIR model with continuous age-structure in the exposed and infectious classes, J. Biol. Dyn., 9 (2015), 73-101.

[25] J.-L. Wang, R. Zhang, T. Kuniya, A note on dynamics of an age-of-infection cholera model, Math. Biosci. Eng., 13 (2016), 227-247.

[26] J.-L. Wang, R. Zhang, T. Kuniya, The dynamics of an SVIR epidemiological model with infection age, IMA J. Appl. Math., 81 (2016), 321-343. 1, 2.3

[27] G. F. Webb, Theory of nonlinear age-dependent population dynamics, Monographs and Textbooks in Pure and Applied Mathematics, Marcel Dekker, Inc., New York, (1985). 1, 2.1

[28] J.-X. Yang, Z.-P. Qiu, X.-Z. Li, Global stability of an age-structured cholera model, Math. Biosci. Eng., 11 (2014), $641-665$. 1 